\title{
Optimized Constellations for Two-Way Wireless Relaying with Physical Network Coding
}

\section{Citation}

Toshiaki, Koike-Akino, Petar Popovski, and Vahid Tarokh. Forthcoming. Optimized constellations for two-way wireless replaying with physical network coding. IEEE Journal of Selected Areas in Communications.

\section{Published Version}

http://ieeexplore.ieee.org/xpl/Recentlssue.jsp?punumber=49

\section{Permanent link}

http://nrs.harvard.edu/urn-3:HUL.InstRepos:2748509

\section{Terms of Use}

This article was downloaded from Harvard University's DASH repository, and is made available under the terms and conditions applicable to Open Access Policy Articles, as set forth at http:// nrs.harvard.edu/urn-3:HUL.InstRepos:dash.current.terms-of-use\#OAP

\section{Share Your Story}

The Harvard community has made this article openly available.

Please share how this access benefits you. Submit a story.

\section{Accessibility}




\title{
Optimized Constellations for Two-Way Wireless Relaying with Physical Network Coding
}

\author{
Toshiaki Koike-Akino ${ }^{\dagger}$, Petar Popovski ${ }^{\ddagger}$, and Vahid Tarokh ${ }^{\dagger}$
}

\begin{abstract}
We investigate modulation schemes optimized for two-way wireless relaying systems, for which network coding is employed at the physical layer. We consider network coding based on denoise-and-forward (DNF) protocol, which consists of two stages: multiple access (MA) stage, where two terminals transmit simultaneously towards a relay, and broadcast (BC) stage, where the relay transmits towards the both terminals. We introduce a design principle of modulation and network coding, considering the superposed constellations during the MA stage. For the case of QPSK modulations at the MA stage, we show that QPSK constellations with an exclusive-or (XOR) network coding do not always offer the best transmission for the $\mathrm{BC}$ stage, and that there are several channel conditions in which unconventional 5-ary constellations lead to a better throughput performance. Through the use of sphere packing, we optimize the constellation for such an irregular network coding. We further discuss the design issue of the modulation in the case when the relay exploits diversity receptions such as multiple-antenna diversity and path diversity in frequency-selective fading. In addition, we apply our design strategy to a relaying system using higher-level modulations of 16QAM in the MA stage. Performance evaluations confirm that the proposed scheme can significantly improve end-to-end throughput for two-way relaying systems.
\end{abstract}

Index Terms-Network coding, two-way relaying, cooperative communications, denoise-and-forward, sphere packing

\section{INTRODUCTION}

Wireless network coding has recently received a lot of attention in research community, although the concept of network coding has been around for almost a decade [2]. This increased interest can be, to a large extent, attributed to the huge gains that are foreseen in cooperative relaying scenarios with two-way or multi-way traffic as addressed in [3-9]. Since Shannon firstly considered a two-way channel in [10], some theoretical investigations on the bidirectional relaying have emerged so far [11]. In this paper, we provide a design strategy of signal constellations and network coding optimized for two-way wireless relaying.

The two-way or bidirectional relaying scenario is depicted in Fig. 1, where the terminal $A$ has traffic to send towards the terminal $B$ and vice versa. The relay $R$ only helps the communications and is neither a traffic source nor a sink. As discussed in [12-15], there are several different protocols in the two-way relaying scenario. In this paper, we focus on a relaying scheme with physical-layer network coding, illustrated in Fig. 1 (c),

$\dagger$ The authors are with the School of Engineering and Applied Sciences, Harvard University, 33 Oxford Street, Cambridge, MA 02138, U.S.A. tel: +1-617-496-7892, fax: +1-617-496-6404, Email: \{koike, vahid\}@seas.harvard.edu. ‡ The author is with Department of Electronic Systems, Aalborg University, Niels Jernes Vej 12, DK-9220 Aalborg, Denmark. Email: petarp@es.aau.dk. This work was partly reported in GLOBECOM [1]. which is referred to as denoise-and-forward (DNF) in this paper, originally introduced by the author in [6]. This scheme is of particular interest as it represents a radical departure from the conventional wireless design and it promises to bring large gains in a communication-theoretic sense, rather than only in information-theoretic sense $[6,14]$.

The DNF scheme consists of two stages. The first stage is termed multiple access (MA) stage, during which the terminals $A$ and $B$ simultaneously transmit to $R$. Due to the half-duplex constraint, neither terminal can receive anything from the other terminal during the MA stage. In the MA stage, the relay $R$ maps the received signals into symbols from a discrete constellation rather than the use of joint decoding [16]. In the second stage, termed broadcast $(B C)$ stage, these symbols are broadcasted to $A$ and $B$. From the fact that $A(B)$ knows its own information a priori, the data from $B(A)$ can be decoded by the broadcasted signal from $R$.

The simplest scenario to illustrate the basic idea of DNF is when the terminals use BPSK modulation in the MA stage. Assume that the channel gains are 1 and the transmitted symbols from $A$ and $B$ are $X_{A}, X_{B} \in\{-1,1\}$. The received signal at $R$ is $Y_{R}=X_{A}+X_{B}+Z_{R}$, where $Z_{R}$ is the noise at the relay. If this MA channel is noiseless $\left(Z_{R}=0\right)$, the possible received signals are $\{-2,0,2\}$. Clearly, if $Y_{R}=0, R$ has residual ambiguity and cannot jointly decode the signals from $A$ and $B$ even in a noiseless case. Nevertheless, $R$ can use the following denoising map: If it receives -2 or 2 , it sends 1 in the $\mathrm{BC}$ stage, while it broadcasts -1 otherwise. When $A$ has sent 1 at the MA stage and it receives -1 from $R$ at the $\mathrm{BC}$ stage, it successfully interprets that $B$ has sent the symbol -1 by using the own data.

The BPSK example described above uses per-symbol denoising, while the information-theoretic approaches [12-14] use per-codeword denoising. Information-theoretic signalling can be often far from practical because it may require the Gaussian-distributed modulations and an infinite packet lengths. In this paper, we investigate per-symbol denoising when practical higher-level modulations such as QPSK and 16QAM are used at the MA stage. The motivation is two-fold: 1) per-symbol denoising is closer to practical communications, as it can be applied for packets of small length and 2) the peculiar operation at the relay gives rise to interesting problems related to the modulation design in terms of choice of the signal constellation and the network coding used at the relay. The originality of the problems stems from the fact that the relay has to use constellation points that should be interpreted differently by each terminal, as dictated by the information that is sent by the terminal during the MA stage. 

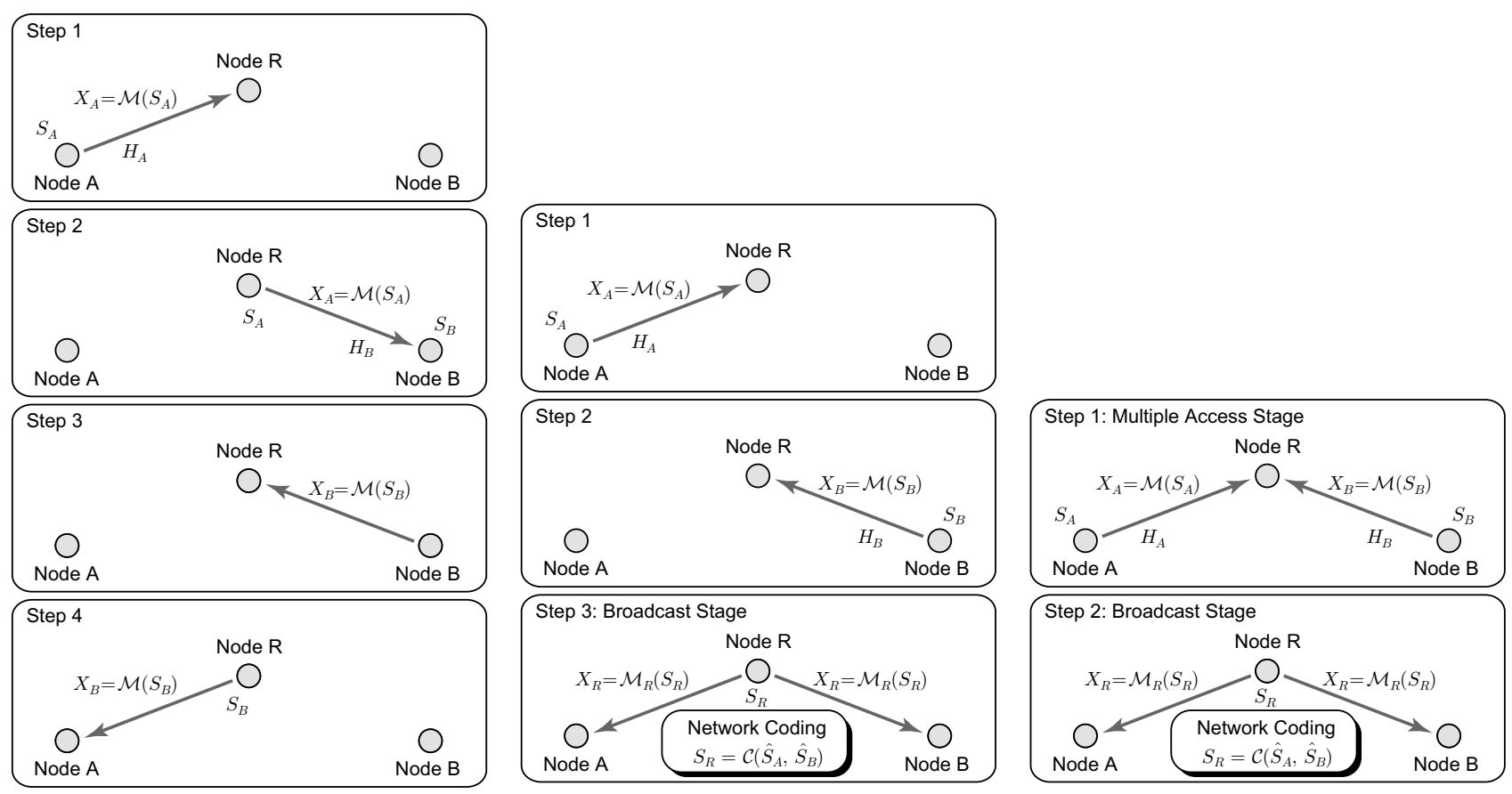

(a)

(b)

(c)

Fig. 1. Two-way relaying systems: (a) conventional 4-stage protocol, (b) 3-stage protocol with network coding, (c) 2-stage protocol with denoising.

The two-stage denoising has a potential to increase system throughput due to its time efficiency, as compared to the other protocols in Figs. 1 (a) and (b). Nevertheless, when the MA stage uses higher-level constellations, the denoising scheme should be more sophisticated, as partly discussed in [6]. This is because the channel coefficients in the MA stage can randomly determine the received constellation in which the two signals are overlapped (i.e., multiple-access interference) at the relay. Meanwhile, the information transmitted in the $\mathrm{BC}$ stage depends on the decision process that is based on the observations that the relay has during the MA stage. In this paper, we tackle the problem of modulation design, and introduce a design principle through investigating the case where both terminals use QPSK in the MA stage. This is certainly not a general approach, but it gives an excellent insight into the relevant issues for modulation designs in two-way relaying. We discuss two different approaches to cope with the problem of modulation design and network coding design for the BC stage. The first approach uses only QPSK constellations for broadcast, but the network coding is optimized based on the observations in the MA stage. The second approach allows the use of unconventional 5-ary modulations in the $\mathrm{BC}$ stage, to further improve reliability. We analyze the proposed schemes in terms of adaptation to the actual channel conditions. An important dividend is that the denoising maps are designed so as to limit the amount of required channel information at the terminals for a practical use. Our simulation results show that the presented approach can improve the throughput performance significantly.

In the second part of the paper, we analyze extensions of our modulation design strategy. We first introduce a simplified version of the code selection algorithm to improve reliability at the BC stage. Next, we extend our design method to the case when larger constellations of 16QAM are used in the MA stage. Finally, we study on the modulation design by considering diversity reception at the relay that exploits multiple-antenna diversity or path diversity in frequencyselective fading channels. The major contributions of this paper are summarized as follows:

- we develop a closest-neighbor clustering method to design network coding for reliable two-way relaying,

- we present well-designed network coding rules for QPSK relaying scenario, some of which require 5-ary cardinality to improve system throughput,

- we optimize a signal constellation for 5-ary network coding by means of sphere packing [17],

- we demonstrate the constellation design for two-way relaying that uses 16QAM at the MA stage,

- we introduce a way to optimize network coding when we exploit diversity reception techniques.

The rest of this paper is organized as follows: In section II, we overview the bidirectional wireless relaying systems using two-stage DNF protocol. In section III, we illustrate a design issue for network coding and propose a design method. We obtain well-designed network codes for a case of QPSK modulation at the MA stage. We then reveal that an irregular 5 -ary network coding is required to improve performance. We optimize a signal constellation of 5QAM for the irregular 5 -ary network coding by making use of sphere packing. 
Next, we evaluate the performance gains of the proposed scheme in bidirectional wireless relaying channels through computer simulations. In the following section IV, we further describe some additional notes to extend the proposed method into a system using higher-level modulations and diversity receptions. Finally, we conclude the paper in section V.

\section{Two-Stage Bidirectional Relaying}

\section{A. Multiple Access (MA) Stage}

Letting $\mathcal{M}$ be a QPSK constellation mapper used at the MA stage, the transmitting signals from $A$ and $B$ are written as $X_{A}=\mathcal{M}\left(S_{A}\right)$ and $X_{B}=\mathcal{M}\left(S_{B}\right)$. Here, $S_{A}$ and $S_{B}$ are digital source data per symbol from $A$ and $B$, respectively. For the case of QPSK, $S_{A}$ and $S_{B}$ are 2-bit binary tuples, i.e., $S_{A}, S_{B} \in \mathbb{Z}_{4}$, where $\mathbb{Z}_{q}=\{0,1, \ldots, q-1\}$ denotes a nonnegative integer set. We assume that the QPSK constellation has unity energy and uses the Gray mapping. The received signal at the relay $R$ over the MA channel is written as

$$
Y_{R}=H_{A} X_{A}+H_{B} X_{B}+Z_{R},
$$

where $H_{A}$ and $H_{B}$ are the channel coefficients from the terminals $A$ and $B$, respectively. Here, $Z_{R}$ is the Gaussian noise with a variance of $\sigma^{2}$. We assume a slow block fading and a perfect channel estimation at the receivers.

\section{B. Denoise-and-Forward (DNF)}

The relay $R$ employs a denoising function to map the received signal $Y_{R}$ into a quantized signal $X_{R}$. We consider a denoising function consisting of a denoising mapper $\mathcal{C}$ and a constellation mapper $\mathcal{M}_{R}$, preceded by the maximumlikelihood (ML) detection. The ML detection performs

$$
\left(\hat{S}_{A}, \hat{S}_{B}\right)=\underset{\left(s_{1}, s_{2}\right) \in \mathbb{Z}_{4} \times \mathbb{Z}_{4}}{\operatorname{argmin}}\left|Y_{R}-\left(H_{A} \mathcal{M}\left(s_{1}\right)+H_{B} \mathcal{M}\left(s_{2}\right)\right)\right|^{2},
$$

to obtain the ML estimates $\hat{S}_{A}$ and $\hat{S}_{B}$. The denoising map $\mathcal{C}$ generates a network-coded data $S_{R}$ from the ML estimates $\hat{S}_{A}$ and $\hat{S}_{B}$; i.e., $S_{R}=\mathcal{C}\left(\hat{S}_{A}, \hat{S}_{B}\right)$. Using the constellation mapper $\mathcal{M}_{R}$, the denoised signal is then given as $X_{R}=\mathcal{M}_{R}\left(S_{R}\right)$. Note that the ML detection is used just for obtaining the quantized version of the received signal, not for joint decoding. The communication throughput through the MA channel is inevitably restricted by the weaker link of the two terminals.

The relaying node $R$ selects the best denoising map out of a well-designed finite mapping book according to the channel condition. For successful decoding, any arbitrary mapping $\mathcal{C}$ must meet the following requirement:

$$
\begin{aligned}
& \mathcal{C}\left(s_{1}, s_{2}\right) \neq \mathcal{C}\left(s_{1}^{\prime}, s_{2}\right) \text { for any } s_{1} \neq s_{1}^{\prime} \in \mathbb{Z}_{4} \text { and } s_{2} \in \mathbb{Z}_{4}, \\
& \mathcal{C}\left(s_{1}, s_{2}\right) \neq \mathcal{C}\left(s_{1}, s_{2}^{\prime}\right) \text { for any } s_{2} \neq s_{2}^{\prime} \in \mathbb{Z}_{4} \text { and } s_{1} \in \mathbb{Z}_{4},
\end{aligned}
$$

which we refer to as exclusive law hereafter. One way to design a denoising map is to select the map so as to correspond to the well-known exclusive-or (XOR) operation as follows

$$
\mathcal{C}\left(S_{A}, S_{B}\right)=S_{A} \oplus S_{B}
$$

where $\oplus$ denotes a bit-wise XOR operation. As it achieves the smallest cardinality 4 , the signal constellation $\mathcal{M}_{R}$ may

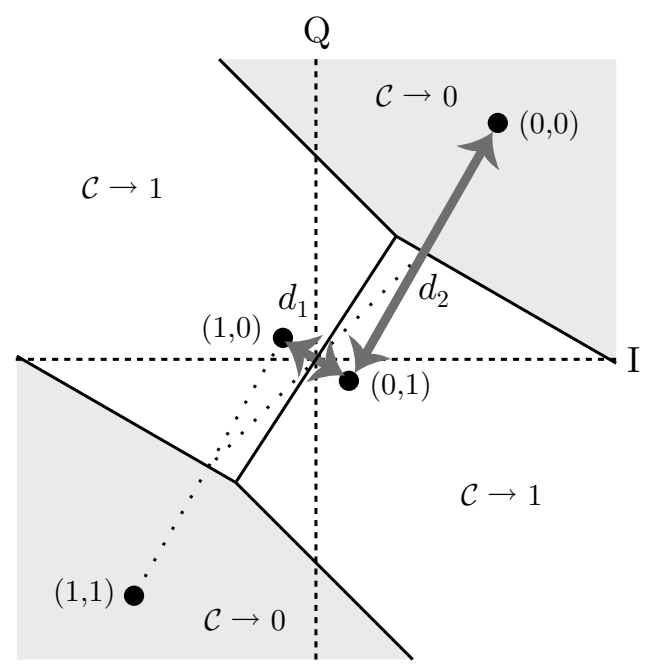

Fig. 2. Received constellation when two BPSK signals are superposed in the MA stage (XOR denoising).

be QPSK in the BC stage just like in the MA stage. However, as we will see later, the channel gains do not always favor such an XOR design for the denoising mapper.

\section{Broadcast (BC) Stage}

In the $\mathrm{BC}$ stage where the denoised signal is broadcasted to $A$ and $B$, the received signals at the terminals $A$ and $B$ are respectively written as

$$
Y_{A}=H_{A} X_{R}+Z_{A}, \quad Y_{B}=H_{B} X_{R}+Z_{B},
$$

where $Z_{A}$ and $Z_{B}$ are the Gaussian noises. For simplicity, we assume the reciprocal channel for both stages, and the identical noise variance $\sigma^{2}$ for $Z_{A}$ and $Z_{B}$. Thanks to the exclusive law, the terminal $A$ can detect the desired data $S_{B}$ by using its own information $S_{A}$ as follows

$$
\hat{S}_{B}^{\prime}=\underset{s \in \mathbb{Z}_{4}}{\operatorname{argmin}}\left|Y_{A}-H_{A} \mathcal{M}_{R}\left(\mathcal{C}\left(S_{A}, s\right)\right)\right|^{2},
$$

assuming the successful forwarding at the relay $R$ so that $\mathcal{C}\left(\hat{S}_{A}, \hat{S}_{B}\right)=\mathcal{C}\left(S_{A}, S_{B}\right)$. In a similar way, the terminal $B$ detects the desired data $S_{A}$ by using its own information $S_{B}$.

\section{Physical Network Coding in DNF}

Compared to the three-stage and four-stage protocols, the two-stage protocol can improve throughput because of the efficiency in the time resource usage [14]. Here, we see the fundamental function of physical-layer network coding in the two-stage protocol. Fig. 2 illustrates the simplest example using the BPSK modulation at the MA stage. This figure shows an instance of the received signal constellation at the relay $R$. Since two BPSK signals are superposed, there are four possible signal points. Using an XOR network coding for the mapping function $\mathcal{C}$ can decrease the cardinality of the denoised symbol from 4-ary to 2-ary. Thus, we only require BPSK modulations for the BC stage to convey combined two packets. This compression function directly translates into a more reliable BC stage, since the constellation transmitted from the relay 
contains fewer points, which, for a fixed transmission power, implies larger Euclidean distances among them.

The physical-layer network coding can improve reliability of denoising at the MA stage as well. As shown in this figure, the minimum distance $d_{1}$ between the signal points $\left(S_{A}, S_{B}\right)=(0,1)$ and $(1,0)$ is quite short. It implies that the joint decode-and-forward (JDF) [14] may not work well because it causes serious decoding errors. Since the relay is not the final destination, DNF uses a denoising map without decoding and error checking. Because the XOR denoising generates the same code word for the adjacent nominal received points, i.e., $\mathcal{C}(0,1)=\mathcal{C}(1,0)=1$, the minimum distance between the different denoising points becomes much larger from $d_{1}$ to $d_{2}$, that improves reliability at the MA stage.

\section{E. Related Works}

Bidirectional (and multi-directional) wireless relaying systems that use network coding have been introduced in $[3,4]$. Those works use the decode-and-forward (DF) scheme, where each terminal transmits to the relay without interference from the other terminal and the packets are combined by XOR for broadcasting. The DF scheme has been applied to a larger network scenario and real-life protocols in $[7,8]$. In $[6,18]$, the amplify-and-forward (AF) bidirectional relaying is introduced, where the terminal nodes simultaneously transmit to the relaying node, and subsequently the relay broadcasts the received signal after amplification. This AF relaying, under the name analog network coding, has been implemented in [9]. The observation, that the relay does not need to decode the received signal, is taken a step further in [15], where DNF is introduced. Independently, identical technique under the name physical-layer network coding (PNC) was introduced in [19]. Several protocols are studied under informationtheoretic framework in [12-14]. The paper in [20] analyzes the optimal broadcast strategy that should be applied by the relay node that has decoded the packets from both nodes. The usage of structured codes and lattices in the scenarios for two-way relaying with per-codeword denoising at the relay has been investigated in [21,22]. The diversity-multiplexing tradeoff has been studied in [23]. Finally, in [24], multipleantenna techniques have been investigated in order to extract the required information from the received noisy packets.

In this paper, we introduce a novel adaptive network coding and a design method of network-coded constellations. Although we only focus on uncoded systems in this paper, our design method can be applied to the systems which use any kind of error-correcting codes between the end nodes, provided that the relay continues to operate at the symbol level without considering a specific error-correction code. In our upcoming work [25], we are reporting trellis-coded schemes where the relay actively uses the error-correction code structure in the denoising operations.

\section{Mapping And Constellation Design}

The denoising map $\mathcal{C}$ should have larger distance profiles to minimize error probability of $\mathcal{C}\left(S_{A}, S_{B}\right) \neq \mathcal{C}\left(\hat{S}_{A}, \hat{S}_{B}\right)$. Likewise, $\mathcal{M}_{R}$ should have larger distance between the constellation points to minimize the error rate that $\mathcal{M}_{R}\left(\mathcal{C}\left(\hat{S}_{A}^{\prime}, S_{B}\right)\right) \neq$
$\mathcal{M}_{R}\left(\mathcal{C}\left(S_{A}, S_{B}\right)\right)$ or $\mathcal{M}_{R}\left(\mathcal{C}\left(S_{A}, \hat{S}_{B}^{\prime}\right)\right) \neq \mathcal{M}_{R}\left(\mathcal{C}\left(S_{A}, S_{B}\right)\right)$. By observing only the BC stage, it is clear that it is better to use as low-level constellations in $\mathcal{M}_{R}$ as possible, since in that case the Euclidean distances among the constellation points are maximized. Since 4-ary modulations are minimal acceptable for the broadcast in our scenario (otherwise there is insufficient information to recover the symbol from the other terminal), we should use QPSK in the BC stage whenever possible. We first discuss 4-ary denoising maps for QPSK modulations. Next, we show that for each particular 4-ary denoising, there are channel conditions which make it unreliable. Then, we propose a design method for $\mathcal{C}$ to improve the distance profiles, with no cardinality constraint for the constellation mapper $\mathcal{M}_{R}$. The result gives us an interesting fact that 5-ary denoising is better for some channel conditions even though it slightly degrades the reliability at the $\mathrm{BC}$ stage. At last, we optimize the constellation $\mathcal{M}_{R}$ for the irregular 5-ary denoising through the use of sphere packing [17].

\section{A. Design Criterion: Pairwise Error Probability}

To optimize denoising maps, we basically aim at minimizing pairwise error probability between different code words at the MA stage because the MA stage dominates system performance rather than the $\mathrm{BC}$ stage due to the presence of multiple-access interference. The squared Euclidean distance between the transmitted data $\left(S_{A}, S_{B}\right)$ and its candidate $\left(\hat{S}_{A}, \hat{S}_{B}\right)$ is written as

$$
d_{\left(S_{A}, S_{B}\right)-\left(\hat{S}_{A}, \hat{S}_{B}\right)}^{2}=\left|H_{A}\right|^{2}\left|\Delta\left(S_{A}, \hat{S}_{A}\right)+\gamma \mathrm{e}^{\jmath \theta} \Delta\left(S_{B}, \hat{S}_{B}\right)\right|^{2},
$$

where we define $\Delta\left(s, s^{\prime}\right)=\mathcal{M}(s)-\mathcal{M}\left(s^{\prime}\right)$. Here, $\gamma$ and $\theta$ are the channel amplitude ratio and the channel phase difference as follows: $H_{B} / H_{A}=\gamma \exp (\jmath \theta)$. Supposed that this data pair is erroneous, more specifically, $\mathcal{C}\left(S_{A}, S_{B}\right) \neq \mathcal{C}\left(\hat{S}_{A}, \hat{S}_{B}\right)$, its pairwise error probability is obtained as

$$
\operatorname{Pr}\left(\left(S_{A}, S_{B}\right) \rightarrow\left(\hat{S}_{A}, \hat{S}_{B}\right)\right)=\frac{1}{2} \operatorname{erfc} \sqrt{\frac{d_{\left(S_{A}, S_{B}\right)-\left(\hat{S}_{A}, \hat{S}_{B}\right)}^{2}}{\sigma^{2}}}
$$

It is known that the total error rate is well approximated by a weighted sum of all the possible pairwise error probabilities. Through this summation, the most dominant factor to determine the overall error probability is the minimum squared distance as follows:

$$
d_{\min }^{2}=\min _{\mathcal{C}\left(S_{A}, S_{B}\right) \neq \mathcal{C}\left(\hat{S}_{A}, \hat{S}_{B}\right)} d_{\left(S_{A}, S_{B}\right)-\left(\hat{S}_{A}, \hat{S}_{B}\right)}^{2} .
$$

In principle, we design a denoising map $\mathcal{C}$ in favor of increasing the minimum distance. Since there are many maps that achieve the same minimum distance, we successively decrease the cardinality by considering the second (third, fourth etc.) minimum distance to improve reliability at the $\mathrm{BC}$ stage.

\section{B. Quaternary Denoising Maps for QPSK Constellation}

To obtain a good 4-ary denoising map, we require some sort of 4-color clustering method and obtain 4 clusters from the $16 \mathrm{ML}$ regions under the exclusive law constraint. Since 


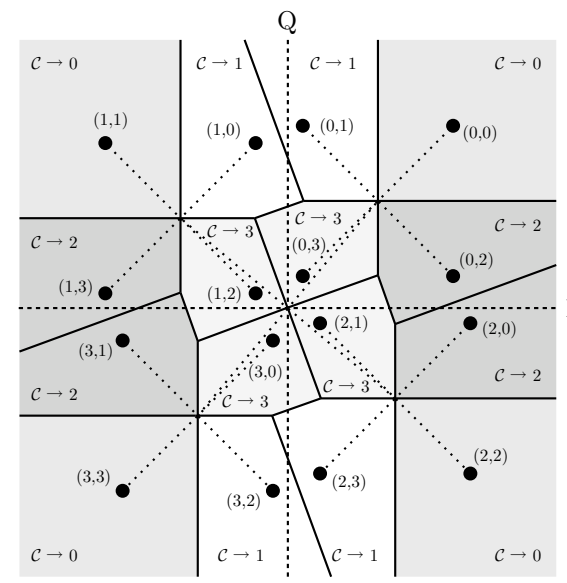

(a) $H_{B} / H_{A} \simeq 1$

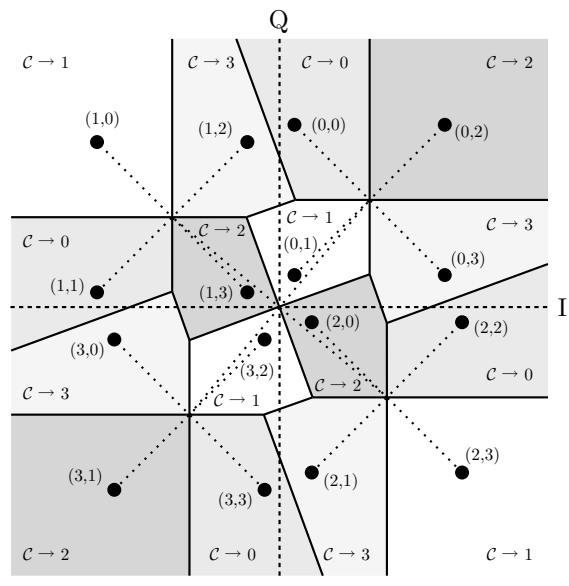

(b) $H_{B} / H_{A} \simeq \jmath$

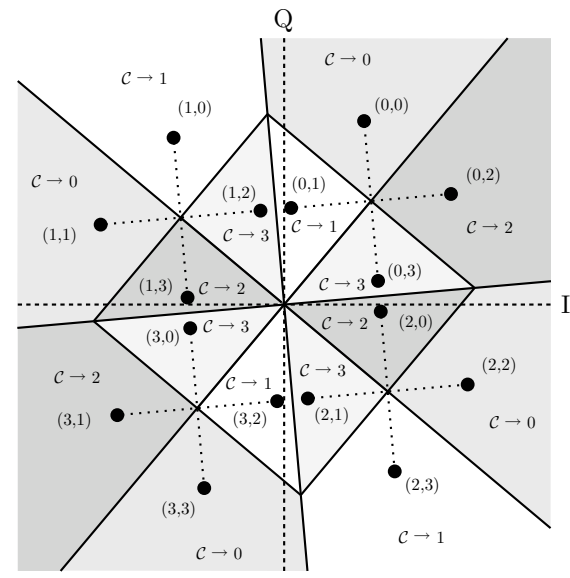

(c) $H_{B} / H_{A} \simeq(1+\jmath) / 2$

Fig. 3. Received signal constellation with XOR denoising map for several channel conditions.

the shape of ML regions highly depends on the channels $H_{A}$ and $H_{B}$, the denoising map should be designed according to the channel condition. For $\theta \simeq 0$, we can use one of the best denoising maps, which is equivalent to the XOR operation in (2) at the digital level. This is illustrated in Fig. 3 (a), that shows a received signal constellation at the relay $R$ for $\theta \simeq 0$ and $\gamma \simeq 1$. This figure shows that the necessary clustering of the closest neighboring regions (e.g., the 4 regions in the center) successfully results from the XOR operation, and it can increase the minimum distance considerably.

However, such a clustering is not applicable to all the possible channel realizations. For example, consider Fig. 3 (b), where $\theta \simeq \pi / 2$ and $\gamma \simeq 1$. We can see that such a $\pi / 2$ signal rotation of $X_{B}$ leads to shorter distance profiles compared to the case in Fig. 3 (a) because any closest-neighbors are not clustered. In this case, a suitable denoising mapper at the relay results in the following binary relation:

$$
\mathcal{C}\left(S_{A}, S_{B}\right)=S_{A} \oplus \mathcal{R}\left(S_{B}\right),
$$

where $\mathcal{R}(s)$ being an anti-rotation operator given as

$$
\mathcal{R}(0)=1, \quad \mathcal{R}(1)=3, \quad \mathcal{R}(2)=0, \quad \mathcal{R}(3)=2 .
$$

The mapping in (8) offers exactly the same distance profiles for $\theta \simeq \pi / 2$ as the mapping in (2) does for $\theta \simeq 0$. In fact, the best denoising map among all the possible 4-ary maps is the pure XOR mapping in (2) for $|\tan \theta|<1$, and the modified XOR mapping in (8) for $|\tan \theta|>1$. An exhaustive search tells us that there are no other better mappings with 4-ary cardinality for any channel condition, in the sense of minimum distance performance.

Considering the convenience of the XOR denoising, one way to utilize it is to adopt a precoding technique that controls the MA output at the relay to achieve $\theta=0$. However, an accurate phase control among all the distributed nodes may be technically difficult for a practical application. Without such a precoding, a significant performance degradation may occur on the border line of $|\tan \theta|=1$, especially for some specific channel conditions referred to as singular points. We illustrate a case of singular points in Fig. 3 (c), where $\theta=\pi / 4$ and $\gamma \simeq 1 / \sqrt{2}$. Shown in this figure, the 4 -ary XOR network coding cannot cluster all the adjacent regions, and the Euclidean distances of some constellation pairs remain very short. Due to the distance shortening, this particular channel condition leads to a severe performance degradation.

The interesting fact is that at this channel condition there are no denoising maps with 4-ary cardinality which can cluster all the closest-neighboring pairs. Due to the exclusive law, the achievable minimum cardinality is 5 , whose map is given as

$$
\mathcal{C}\left(s_{1}, s_{2}\right)= \begin{cases}0, & \text { for }\left(s_{1}, s_{2}\right)=\{(0,0),(1,1),(2,2),(3,3)\}, \\ 1, & \text { for }\left(s_{1}, s_{2}\right)=\{(0,3),(2,0),(3,1)\}, \\ 2, & \text { for }\left(s_{1}, s_{2}\right)=\{\underline{(0,1),(1,2)},(2,3)\}, \\ 3, & \text { for }\left(s_{1}, s_{2}\right)=\{\underline{(3,2),(2,1)},(1,0)\}, \\ 4, & \text { for }\left(s_{1}, s_{2}\right)=\{\underline{(1,3),(3,0)},(0,2)\} .\end{cases}
$$

The above denoising map can successfully make clusters for all the closest neighbors; more specifically, the underlined four pairs, which are the closest neighbors in Fig. 3 (c), are each mapped into the same code word. It is of great importance to cluster these four pairs in order to avoid distance shortening: This rule necessitates larger cardinality due to the exclusive law. Hence, to improve reliability, we need to allow the use of unconventional modulations, such as 5-ary constellations.

\section{Mapping Design Method: Closest-Neighbor Clustering}

Motivated by the previous observation, we propose a mapping design method that is not constrained by the minimum cardinality of 4 , while achieving large distance profiles. The proposed method is a kind of closest-neighbor clustering algorithms, taking the exclusive law into consideration; it successively makes a cluster for the closest pair only if it satisfies the exclusive law. It is described in Algorithm 1.

This algorithm provides one best code which has the largest distance profile, given a channel condition $(\gamma$ and $\theta)$. Since 

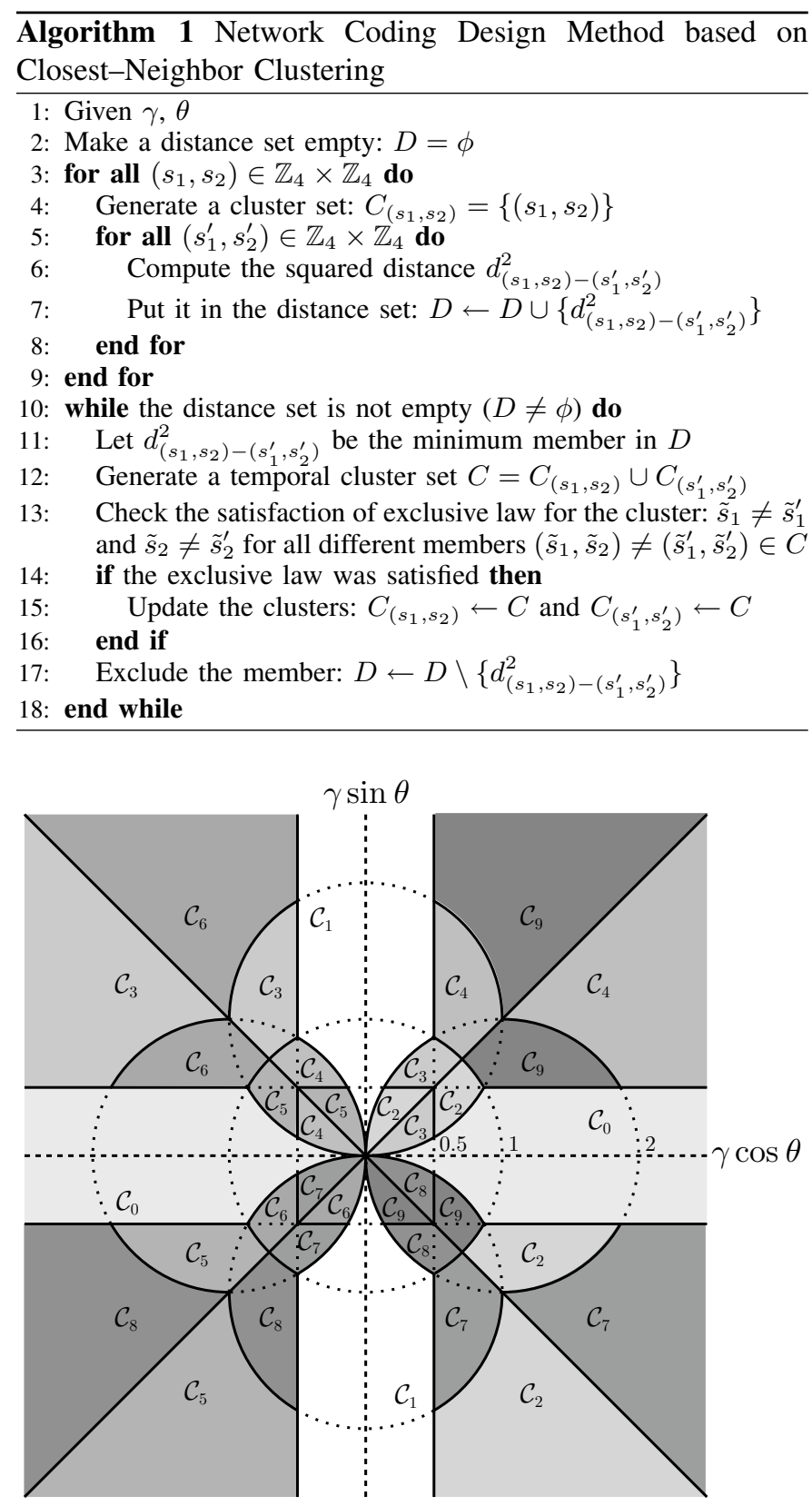

Fig. 4. Adaptive selection of denoising maps given by the proposed design method allowing 5-ary network codes according to the channel ratio $H_{B} / H_{A}=\gamma(\cos \theta+\jmath \sin \theta)$.

different best code may be required at a different condition, we should list up all the best codes for all the possible channel conditions, i.e., whole the area in $0 \leq \gamma \leq \infty$ and $0 \leq \theta<2 \pi$. To do so, we quantize the area into a large number of channel realizations, and perform the closest-neighbor clustering to yield the best code for each channel conditions.

Table I lists all the best denoising maps given by the proposed algorithm. Ten best maps can be obtained in total; $\mathcal{C}_{0}$ and $\mathcal{C}_{1}$ are 4 -ary maps $\left(\mathcal{C}_{0}\right.$ is in $(2)$ and $\mathcal{C}_{1}$ is in $\left.(8)\right)$, while the others have 5 -ary cardinality $\left(\mathcal{C}_{2}\right.$ is in $\left.(10)\right)$. Each denoising map represents a particular type of physical-layer network coding. The relaying node should adaptively select a proper map that offers the best performance depending on the channel condition; which we refer to as adaptive network coding in this paper. Fig. 4 shows the adaptive selection rule for those maps to be assigned according to the actual channel ratio $H_{B} / H_{A}$ observed at the relay $R$. We can see that the 4-ary denoising maps are used for $\theta \simeq m \pi / 4$ where $m \in \mathbb{Z}_{4}$, whereas the 5ary denoising maps are needed for $\theta \simeq(2 m+1) \pi / 8$. Such a 5-ary denoising improves reliability for denoising at the MA stage by avoiding distance shortening at around singular points even though it slightly sacrifices the reliability at the BC stage due to the increased cardinality.

It is worth noting that there are no better codes for morethan 5-ary codes in the sense of the pairwise error probability at the MA stage even if we perform the brute-force full searching. Although there exist a lot of other network codes which can avoid a specific distance shortening, those do not appear useful. This is because such codes cannot offer better performance than the codes in Table I at the MA stage and can degrade the performance at the $\mathrm{BC}$ stage due to the larger cardinality of the signalling constellation.

\section{Minimum Distance Performance}

We confirm the significant advantage of the proposed design method in achievable distance performance. Fig. 5 plots the squared minimum distance as a function of $H_{B} / H_{A}=$ $\gamma \exp (\jmath \theta)$. The squared distance is normalized by $2\left|H_{A}\right|^{2}$, which comes from the squared minimum distance of the QPSK constellation multiplied by the channel gain from the terminal $A$. Figs. 5 (a), (b) and (c) show the distance performance achieved by the pure XOR denoising (1 code), the adaptive 4-ary denoising ( 2 codes), and the optimized denoising (10 codes), respectively. As shown in Fig. 5 (a), the minimum distance can fall into zero for some occasional channel conditions unless we adopt adaptive network coding. Then, we proposed the first approach, i.e., 4-ary denoising, which adaptively switch the 4-ary network coding between the pure XOR operation and the modified XOR operation. As shown in Fig. 5 (b), this scheme is beneficial to remove two singular points at around $H_{B} / H_{A}= \pm$ J. We discovered that 5-ary network coding is needed to further eliminate the other eight singular points. When we use the second approach, i.e., 5-ary denoising, which adaptively use the best eight 5ary network codes together with two 4-ary network codes, all undesirable singular points can be deleted and the distance performance is drastically improved as shown in Fig. 5 (c). Note that the distance shortening at $H_{B} / H_{A} \simeq 0$ is inevitable.

\section{E. Constellation Design for Irregular Quintary Denoising}

Since some denoising maps presented in Table I have 5-ary cardinality, we need 5-ary modulations such as 5PSK for $\mathcal{M}_{R}$ at the BC stage. Note that the label $S_{R}=0$ is more probable $(4 / 16)$ than the others $(3 / 16)$. We use sphere packing approach [17] to design the modulation $\mathcal{M}_{R}$ for such an irregular constellation. The sphere packing arranges the constellation points in favor of maximizing the Euclidean distances so that all spheres centered at the signal points are not overlapped under a certain energy constraint. Because of the nonuniform 
TABLE I

Best Denoising Maps Given by the Proposed Design Method

\begin{tabular}{|c|c|c|c|c|c|c|c|c|c|c|c|c|c|c|c|c|c|}
\hline & $(0,0)$ & $(0,1)$ & $(0,2)$ & $(0,3)$ & $(1,0)$ & $(1,1)$ & $(1,2)$ & $(1,3)$ & $(2,0)$ & $(2,1)$ & $(2,2)$ & $(2,3)$ & $(3,0)$ & $(3,1)$ & $(3,2)$ & $(3,3)$ & Cardinality \\
\hline $\mathcal{C}_{0}$ & 0 & 1 & 2 & 3 & 1 & 0 & 3 & 2 & 2 & 3 & 0 & 1 & 3 & 2 & 1 & 0 & 4 \\
\hline $\mathcal{C}_{1}$ & 1 & 3 & 0 & 2 & 0 & 2 & 1 & 3 & 3 & 1 & 2 & 0 & 2 & 0 & 3 & 1 & 4 \\
\hline $\mathcal{C}_{2}$ & 0 & 2 & 4 & 1 & 3 & 0 & 2 & 4 & 1 & 3 & 0 & 2 & 4 & 1 & 3 & 0 & 5 \\
\hline $\mathcal{C}_{3}$ & 3 & 2 & 0 & 1 & 0 & 1 & 2 & 4 & 1 & 3 & 4 & 0 & 4 & 0 & 3 & 2 & 5 \\
\hline $\mathcal{C}_{4}$ & 2 & 1 & 0 & 4 & 0 & 4 & 3 & 2 & 3 & 2 & 1 & 0 & 1 & 0 & 4 & 3 & 5 \\
\hline $\mathcal{C}_{5}$ & 2 & 1 & 3 & 0 & 1 & 4 & 0 & 2 & 3 & 0 & 1 & 4 & 0 & 2 & 4 & 3 & 5 \\
\hline $\mathcal{C}_{6}$ & 1 & 4 & 2 & 0 & 4 & 2 & 0 & 3 & 2 & 0 & 3 & 1 & 0 & 3 & 1 & 4 & 5 \\
\hline $\mathcal{C}_{7}$ & 1 & 0 & 2 & 3 & 4 & 2 & 1 & 0 & 0 & 4 & 3 & 1 & 2 & 3 & 0 & 4 & 5 \\
\hline $\mathcal{C}_{8}$ & 4 & 0 & 1 & 2 & 2 & 3 & 4 & 0 & 0 & 1 & 2 & 3 & 3 & 4 & 0 & 1 & 5 \\
\hline $\mathcal{C}_{9}$ & 0 & 3 & 1 & 2 & 2 & 0 & 4 & 1 & 4 & 1 & 0 & 3 & 3 & 4 & 2 & 0 & 5 \\
\hline
\end{tabular}

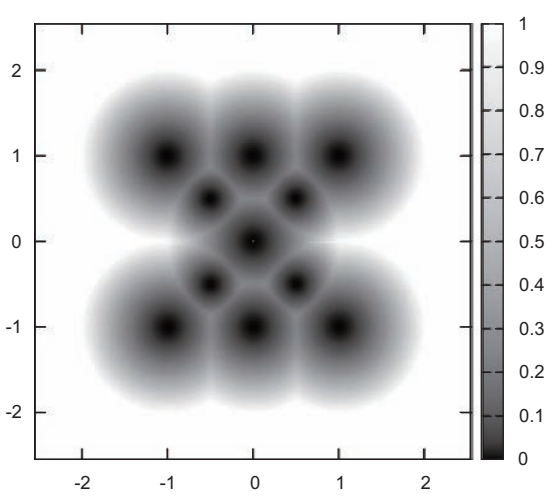

(a) Pure XOR denoising (1 code)

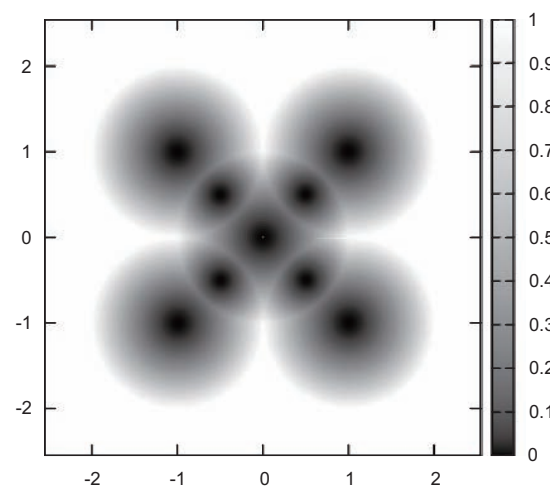

(b) 4-ary denoising (2 codes)

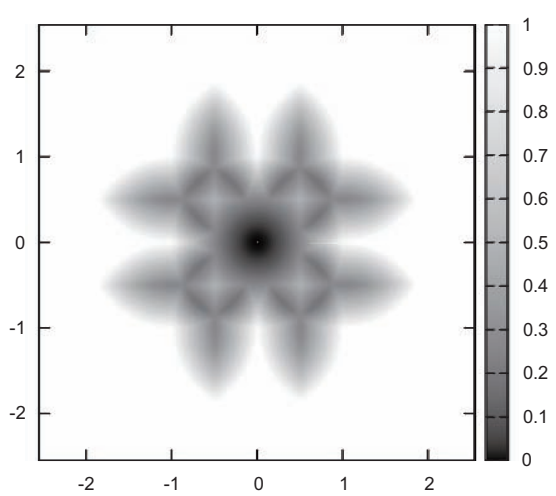

(c) Optimized denoising (10 codes)

Fig. 5. Normalized minimum square-distance as a function of $H_{B} / H_{A}=\gamma(\cos \theta+\jmath \sin \theta)$. x-axis: $\gamma \cos \theta$, y-axis: $\gamma \sin \theta$, $\mathrm{z}$-axis mapped with palette: $d_{\min }^{2} / 2\left|H_{A}\right|^{2}$.

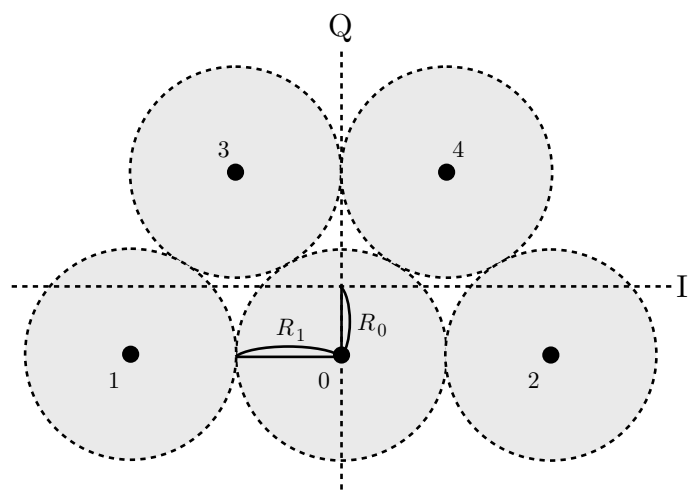

Fig. 6. 5QAM optimized by sphere packing approach.

a priori probabilities, the radii should be non-identical and dependent on the received signal-to-noise power ratio (SNR).

Making use of sphere packing, we derive an asymptotically optimized 5QAM constellation depicted in Fig. 6. In order to have unity energy in average, the sphere radius $R_{1}$ is $8 / \sqrt{165}$, and the offset $R_{0}$ is $3 / \sqrt{55}$. The detailed derivation of the designed 5QAM can be found in Appendix. Although this optimized modulation is given by assuming an infinite SNR, it is confirmed that 5QAM with variable radii, adaptively optimized as a function of SNR, offers almost no advantage in comparison to the constellation in Fig. 6 for any reasonable SNR region. Considering the minimum squared distance
$(2-2 \cos (2 \pi / 5)$ for 5PSK, 2 for QPSK, and $256 / 165$ for $5 \mathrm{QAM}$ ), we may expect $0.5 \mathrm{~dB}$ gain from 5 PSK and $1.1 \mathrm{~dB}$ loss from QPSK. The $1.1 \mathrm{~dB}$ loss may be a drawback of 5-ary denoising against 4-ary denoising at the BC stage. However, 5-ary denoising can outperform 4-ary denoising in end-to-end throughput performance because it can avoid distance shortening that occurs due to the unfavorable mixing (interference) of the signals at the MA stage.

Another remark is in place: In our approach, the ML estimates $\hat{S}_{A}$ and $\hat{S}_{B}$ are obtained independently of the denoising mapper and the constellation mapper. Therefore, for example, the ML regions in Fig. 2 coincide with the Voronoi regions. However, once we fix the denoise mapping $\mathcal{C}$ and the constellation mapping $\mathcal{M}_{R}$, the quantizing decisions at the relay in the MA stage should be modified from the Voronoi regions to the exact ML regions. Nevertheless, we do not expect any significant difference in the performance, and a closer study of this issue is out of the scope for this paper.

\section{F. End-to-End Throughput Evaluations}

We next evaluate the proposed denoising scheme in an end-to-end throughput. We assume that the channels $H_{A}$ and $H_{B}$ follow a frequency-flat Nakagami-Rice fading with a certain Rician factor. The Rician factor is a power ratio between stationary and scattered-wave components. We define an average SNR as $\mathbb{E}\left[\left|H_{A}\right|^{2}+\left|H_{B}\right|^{2}\right] / 2 \sigma^{2}$, where we choose the channel power ratio between $\mathbb{E}\left[\left|H_{A}\right|^{2}\right]$ and $\mathbb{E}\left[\left|H_{B}\right|^{2}\right]$ from 


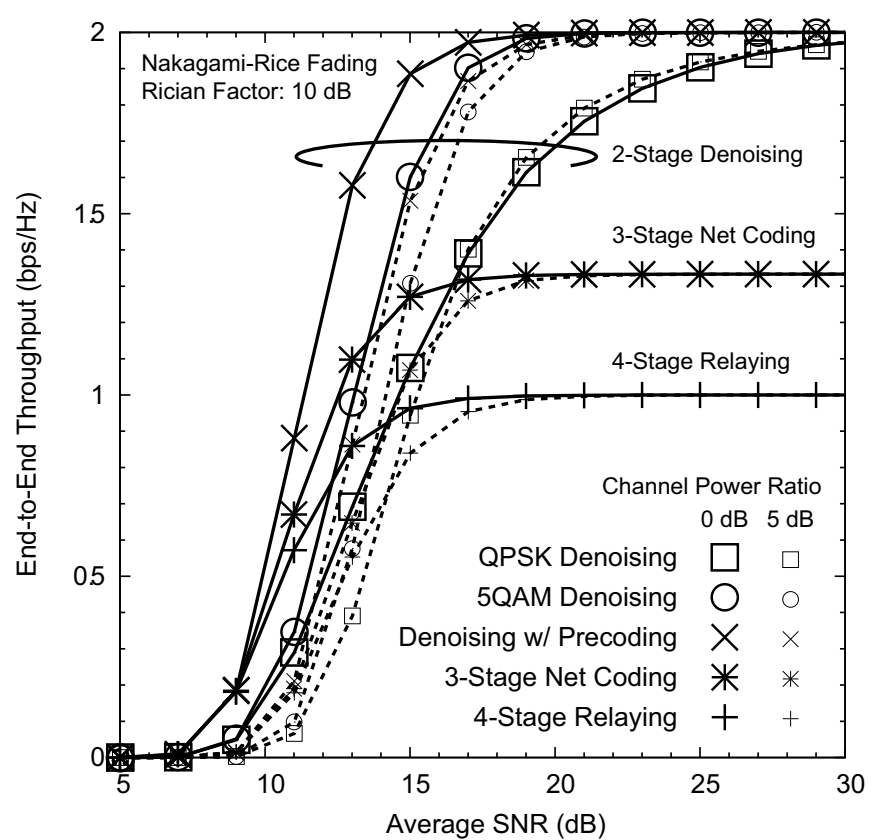

(a) $10 \mathrm{~dB}$ Rician factor

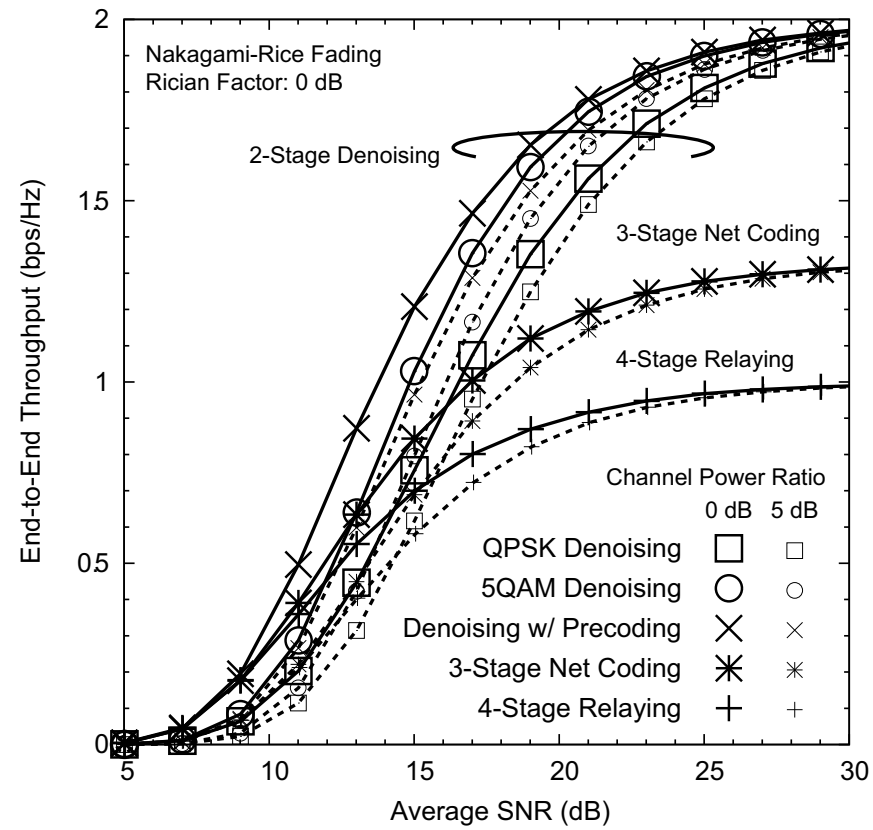

(b) $0 \mathrm{~dB}$ Rician factor

Fig. 7. End-to-end throughput as a function of average SNR in Nakagami-Rice fading channels. (QPSK used at the MA stage)

two values $0 \mathrm{~dB}$ and $5 \mathrm{~dB}$. The notation $\mathbb{E}[\cdot]$ is an expectation function. The data packet has 256 symbols. All the terminals are not phase-synchronized. The nodes $A$ and $B$ do not require any channel state information prior to the transmission.

At the MA stage, the nodes $A$ and $B$ use uncoded QPSK modulations. The relay $R$ adaptively selects the best denoising map out of the mapping book in Table I according to the observed channel ratio $H_{B} / H_{A}$ as in Fig. 4. When 4-ary map is chosen, QPSK signal is broadcasted. Unless otherwise, 5QAM in Fig. 6 is used. In order to announce the selected map towards $A$ and $B$, the map index (at most 4 bits from 0 to 9 in Table I) is inserted into the packet header for the BC stage.

Figs. 7 (a) and (b) show an end-to-end throughput for a Rician factor of $10 \mathrm{~dB}$ and $0 \mathrm{~dB}$, respectively. For comparison, we present the curves of XOR denoising with and without precoding (to achieve $\theta=0$ ). We also plot the curves of the three-stage XOR network coding and the four-stage bidirectional relaying. In the three-stage protocol, the nodes $A$ and $B$ transmit the own packet at the first stage and the second stage, respectively. If no error occurred for both packets, the relay $R$ broadcasts the XOR network-coded packets using QPSK at the last stage. If both packets were erroneous, these packets cannot be relayed, leading to a packet failure. Otherwise, just one successful packet is forwarded to the desired terminal at the last stage. It is assumed that the direct channel between the terminals $A$ and $B$ has a negligibly small SNR, that leads to no available overhearing gains $[12,13]$.

From these figures, it is confirmed that two-stage denoising has a significant advantage compared to the conventional protocols because of the time efficiency. Although a precoding technique offers the best performance, it requires an accurate phase control amongst the distributed terminals at the MA stage. Without precoding, the 4-ary denoising suffers from a severe performance degradation due to distance shortening occurred at singular points. Meanwhile, the denoising function allowing 5QAM considerably outperforms the one that uses only QPSK by $5.6 \mathrm{~dB}$ and $2.8 \mathrm{~dB}$ at $90 \%$ throughput achievement $(1.8 \mathrm{bps} / \mathrm{Hz})$ in Figs. 7 (a) and (b), respectively. The performance improvement by 5 -ary denoising becomes small at a low Rician factor. The chief reason is that the achievable throughput is dominated by the Rayleigh-faded poor channel (which cannot be improved by any network coding) rather than by the multiple-access interference at the first MA stage.

Fig. 8 shows the performance curves of the required SNR for achieving $90 \%$ throughput as a function of Rician factor for various 2-stage denoising schemes (the channel power ratio is $0 \mathrm{~dB}$ ). In this figure, we also present the performance of nonadaptive XOR denoising for reference. One can see that the pure XOR network coding suffers from a severe performance degradation without precoding. The adaptive 4-ary denoising with best 2 codes can decrease the required SNR by $2.5 \mathrm{~dB}$ for a high Rician factor, and by $1.0 \mathrm{~dB}$ for a zero Rician factor (i.e., for Rayleigh fading channels). The adaptive 5-ary denoising can further improve performance especially for a high Rician factor. Even though its performance improvement is only $2.5 \mathrm{~dB}$ for Rayleigh fading channels, it closely approaches the ideal performance which is achieved by precoding. It can be observed that the performance at $0 \mathrm{~dB}$ Rician factor (when Rician factor equals 1 ) is not significantly different from that of Rayleigh fading channels (when Rician factor equals 0); the performance gap is around $1 \mathrm{~dB}$.

If the terminals have large frequency offset, the best code se- 


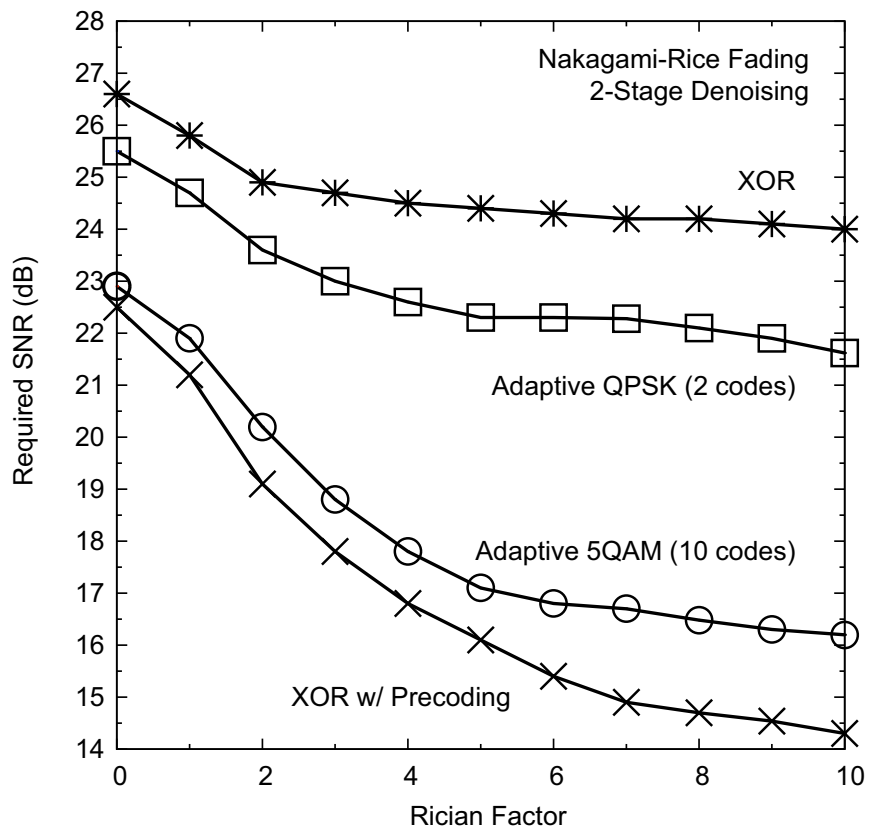

Fig. 8. Required SNR to achieve $1.8 \mathrm{bps} / \mathrm{Hz}$ throughput as a function of Rician factor. (2-stage denoising, $0 \mathrm{~dB}$ channel power ratio)

lection should be frequently changed in a packet. It arises also in a very fast fading channel. Without changing the best code selection throughout a packet in such time-varying channels, the 5-ary denoising suffers from performance degradations and the advantage over the 4-ary denoising can shrink. To suppress the effect of channel variation, the packet length should be decreased in practice.

\section{FURTHER DISCUSSION}

In this section, we extend the basic concepts presented so far. First, we devise a simplified criterion for adaptive selection of the constellation and mapping. Next, we illustrate the design approach when the terminal nodes use higherlevel constellation (16QAM). Finally, we extend the discussion to a relaying system that exploits diversity reception (e.g. multiple-antenna diversity or path diversity in frequencyselective fading channels).

\section{A. Simplified Minimum-Distance Criterion}

In this section, we explore the possible simplification of the adaptive network code selection to decrease the total number of required codes. As listed in Table I, there are ten best network codes derived by the closest-neighbor clustering method. If we adaptively use them at a proper channel condition as in Fig. 4, they can achieve the largest distance profile. It first chooses the subset of possible mappings (i.e. the network codes) that have the largest value of the minimum squared distance, as it is the dominant factor to determine error rate performance. We further reduce the subset by keeping only the network codes that yield the largest value of the secondminimum distance. We continue in analogous way for the third minimum distance etc.

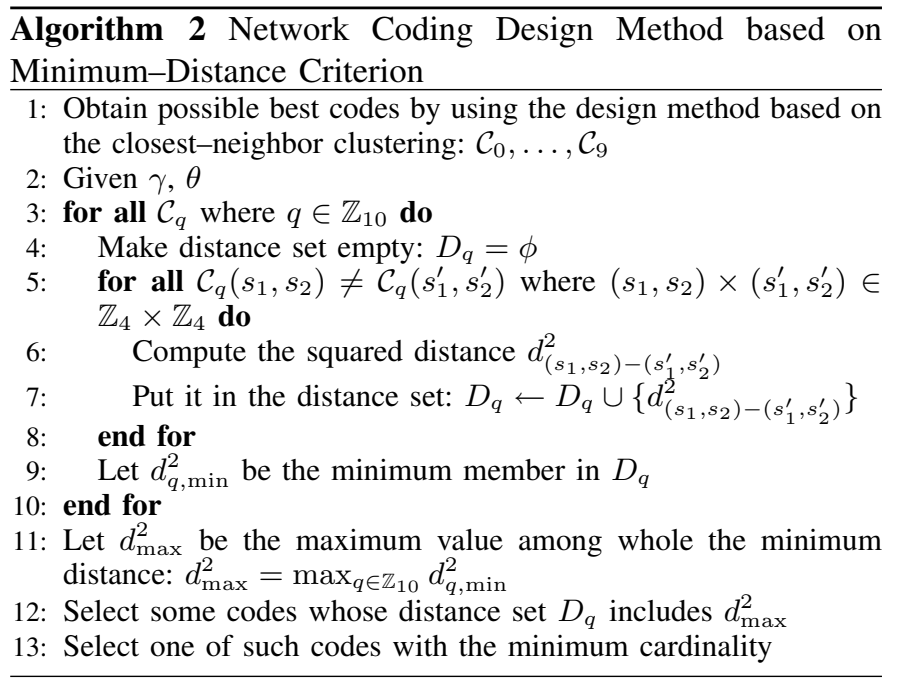

From Table I and Fig. 4, we can conclude that the number of network codes to be used in the adaptive selection is relatively large. This number can be reduced by recognizing that the performance is dominated by the minimum Euclidean distance, especially at a high SNR. By focusing on maximizing the minimum distance only, the required number of the best network codes is decreased from ten to six; more specifically, four codes $\left\{\mathcal{C}_{2}, \mathcal{C}_{4}, \mathcal{C}_{6}, \mathcal{C}_{8}\right\}$ or $\left\{\mathcal{C}_{3}, \mathcal{C}_{5}, \mathcal{C}_{7}, \mathcal{C}_{9}\right\}$ are not needed any more. It is illustrated in Figs. 9 (a) and (b), both of which show the same received signal constellation for $\gamma \simeq 1 / \sqrt{2}$ and $\theta \simeq \pi / 4$. In this channel condition, the best network coding $\mathcal{C}_{2}$ can offer the largest distance profile. The former figure shows the network coding $\mathcal{C}_{2}$ and the latter $\mathcal{C}_{3}$. As shown in these figures, no matter which codes we use, the minimum distance $d_{1}$ is equivalent. The benefit of the best code $\mathcal{C}_{2}$ is a slight increase of the eighth minimum distance $d_{2}$ in comparison to $\mathcal{C}_{3}$. Therefore, the overall performance difference between $\mathcal{C}_{2}$ and $\mathcal{C}_{3}$ may be quite small in this channel condition. In consequence, keeping the focus only on the minimum distance, we can exclude four network codes from the ten best codes.

In addition, we should use 4-ary denoising for several channel conditions instead of 5-ary one because the available minimum distances are identical. This is explained in Fig. 9 (c), which shows the received constellation for $\gamma \simeq 1 / 2 \sqrt{2}$ and $\theta=\pi / 4$ with the best code $\mathcal{C}_{2}$. In this case, the minimum distance $d_{1}$ between $(0,0)$ and $(0,1)$ cannot be clustered by any arbitrary network coding due to the exclusive law. The best 5-ary network coding makes clusters to increase the second minimum distance $d_{2}$, e.g., between $(0,1)$ and $(1,2)$. However, we should use 4-ary coding in this case because it offers the minimum cardinality without changing the minimum distance. From the observations above, we propose a modified design strategy based on the minimum-distance criterion, that is summarized in Algorithm 2.

Using this simplified design method, we obtain a modified selection rule as shown in Fig. 10. Comparing with the original selection rule in Fig. 4, we can see that the required number of the network codes is reduced from ten to six and that the required regions for 5-ary denoising are narrowed. Even the area used for the modified XOR denoising $\mathcal{C}_{1}$ can be 


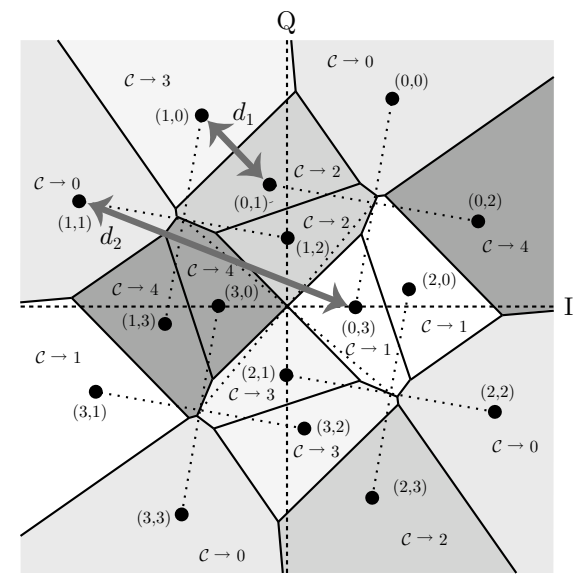

(a) $H_{B} / H_{A} \simeq(1+\jmath) / 2$ with $\mathcal{C}_{2}$

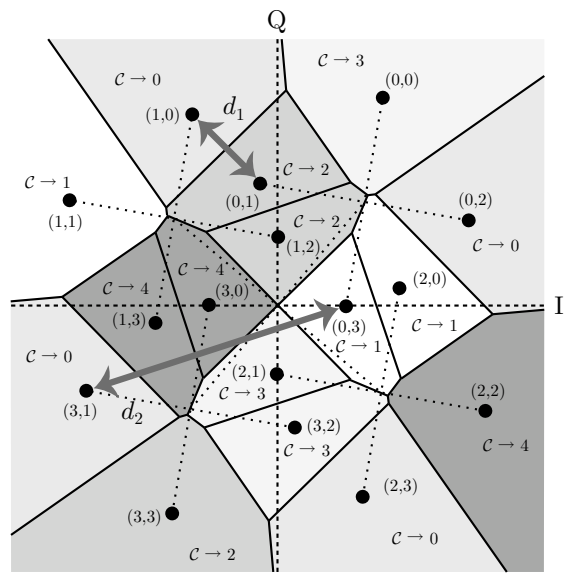

(b) $H_{B} / H_{A} \simeq(1+\jmath) / 2$ with $\mathcal{C}_{3}$

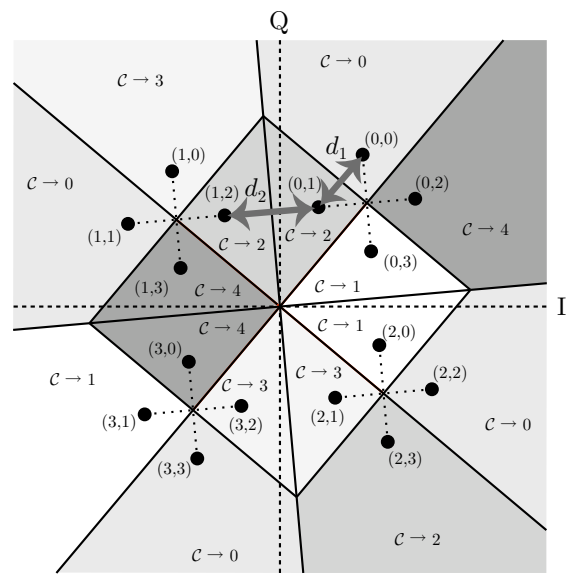

(c) $H_{B} / H_{A} \simeq(1+\jmath) / 4$ with $\mathcal{C}_{2}$

Fig. 9. Received signal constellation with different denoising map for several channel conditions.

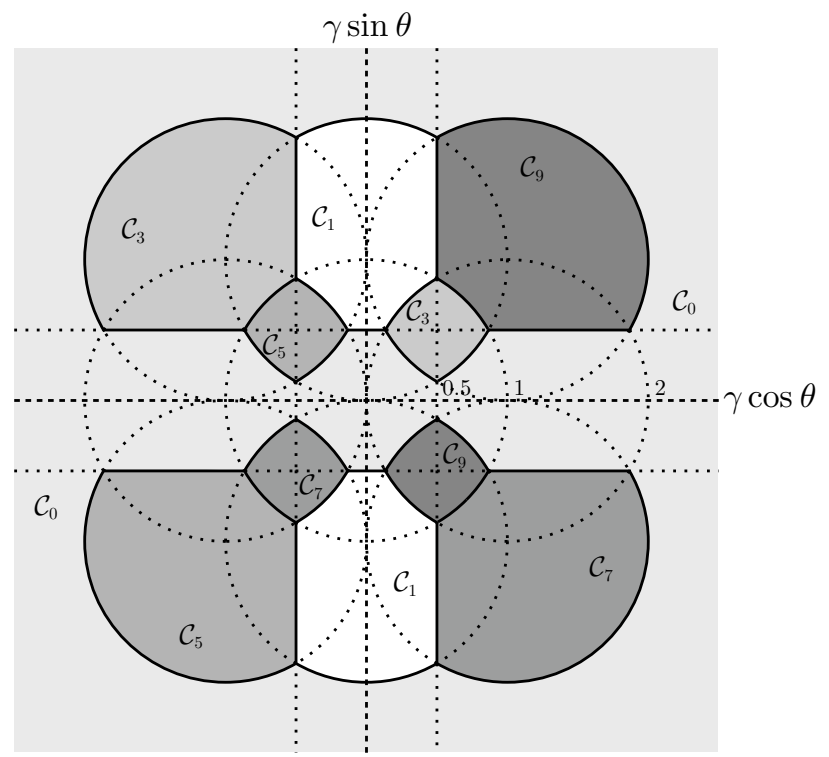

Fig. 10. Simplified adaptive selection of denoising maps given by the proposed design method based on the minimum-distance criterion according to the channel ratio $H_{B} / H_{A}=\gamma(\cos \theta+\jmath \sin \theta)$.

shrunk by the minimum-distance criterion. As a result, the usefulness of the pure XOR denoising $\mathcal{C}_{0}$ grows significantly. The derived selection rule in Fig. 10 is associated with the minimum distance performance depicted in Fig. 5 (a), but the singular points are avoided by the adaptive code selection.

\section{B. 16QAM Bidirectional Wireless Relaying}

For illustration purposes, here we show a result of adaptive network coding for a system that uses a higher-level modulation (16QAM) during the MA stage. For such higher constellations, we can find the significant impact of the simplified design criterion based on the minimum distance, as follows: We obtain more than 18,000 best codes by using the design method based on the closest-neighbor clustering, while the minimum-distance criterion can reduce the required number to approximately 400 . The cardinality of the derived codes spans from 16 to 29. Among the best 400 network codes, there are sixteen codes whose cardinality are 16-ary alphabet size, which is the minimal acceptable value in this case.

In Fig. 11, we show the minimum squared distance as a function of a channel condition $H_{B} / H_{A}=\gamma \exp (\jmath \theta)$; figures (a), (b) and (c) are given by the pure XOR operation, the 16ary denoising with 16 codes, and the optimized denoising with approximately 400 codes, respectively. The squared distance is normalized by $0.4\left|H_{A}\right|^{2}$ which denotes the squared minimum distance of 16QAM constellation multiplied by the channel gain from the terminal $A$. Shown in Fig. 11 (a), distance shortening occurs so frequently and the pure XOR network coding does not work well. Notice that the minimum squared distance cannot reach larger-than $0.3 \times 0.4\left|H_{A}\right|^{2}$. In contrast, Fig. 11 (b) illustrates that the 16-ary denoising with adaptive 16 codes achieves the maximum value $1.0 \times 0.4\left|H_{A}\right|^{2}$ for certain channel conditions; e.g., $\gamma \in\{1.0,2.0\}$ and $\theta=m \pi / 2$ for $m \in \mathbb{Z}_{4}$. Analogously to the QPSK case, it suggests that the 16-ary denoising can be useful if precoding is applicable, such that $H_{B} / H_{A}$ is controlled. However, we can see that there are many singular points left and, again, this problem can be resolved by having the relaying node use irregular modulations at the $\mathrm{BC}$ stage, whose cardinalities are larger than 16. As in Fig. 11 (c), if it is possible to use all the 400 best codes adaptively, we can expect a significant performance improvement because the optimized denoising successfully removes the singular points. Note that, once the relay decides which of the 400 network codes to use, it needs to add only 9 bits in the header of the broadcasting packet.

Provided that we can perform precoding to obtain $\theta=0$, there are seven best codes, all of which have the minimum cardinality of 16 . These codes should be adaptively used for $\gamma \leq 3 / 8,3 / 8 \leq \gamma \leq 3 / 5,3 / 5 \leq \gamma \leq 3 / 4,3 / 4 \leq \gamma \leq 4 / 3$, $4 / 3 \leq \gamma \leq 5 / 3,5 / 3 \leq \gamma \leq 8 / 3$, and $8 / 3 \leq \gamma$, respectively. 


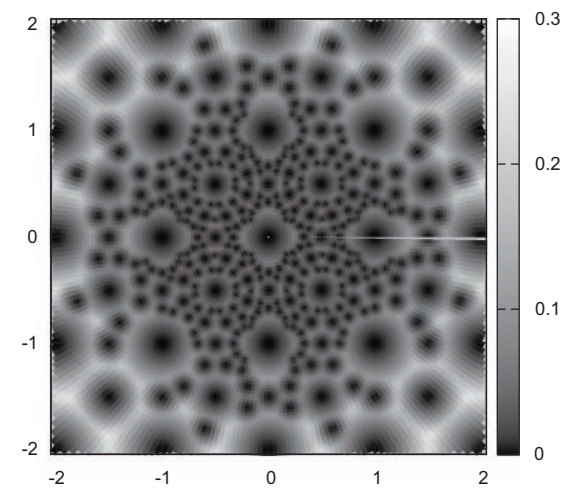

(a) Pure XOR denoising (1 code)

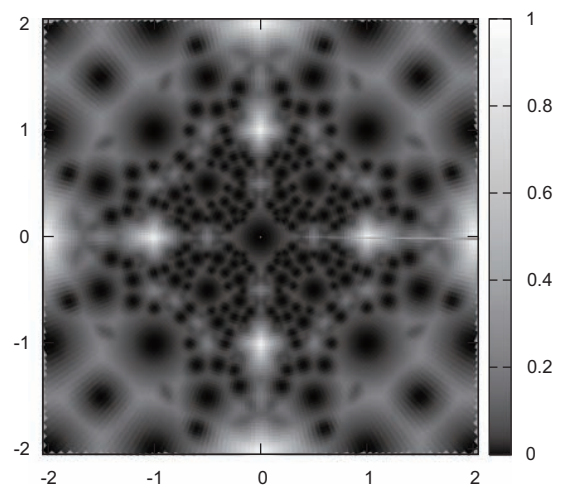

(b) 16-ary denoising (16 codes)

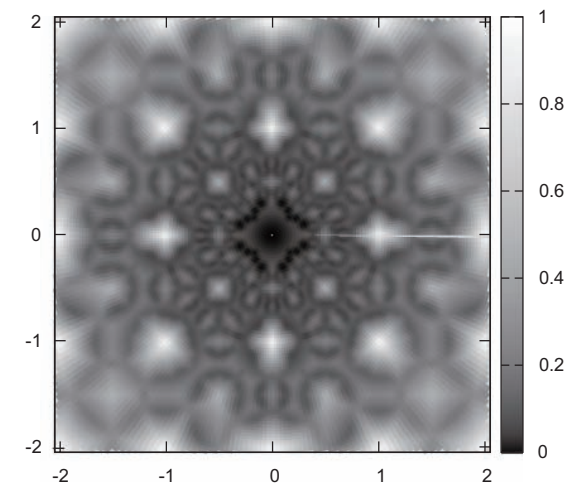

(c) Optimized denoising (approx. 400 codes)

Fig. 11. Normalized minimum square-distance as a function of $H_{B} / H_{A}=\gamma(\cos \theta+\jmath \sin \theta)$ for $16 \mathrm{QAM}$ bidirectional relaying. $\mathrm{x}$-axis: $\gamma \cos \theta$, y-axis: $\gamma \sin \theta, \mathrm{z}$-axis mapped with palette: $d_{\min }^{2} / 0.4\left|H_{A}\right|^{2}$.

As mentioned before, because the 16-ary denoising performs well for $\gamma \in\{0.5,1.0,2.0\}$ and $\theta=0$ where we achieve the maximum value of the minimum distance, it is better to use an amplitude control as well as a phase control for a precoding technique. Clearly, for even higher constellations, the required number of codes for adaptive network coding grows immensely. In general, very high constellations are used if the channel state information is available at the transmitter, which implies that the precoding might be feasible for the scenario intended for large signalling constellations.

\section{Adaptive Network Coding with Diversity Reception}

In this section, we investigate on the design implications in diversity receptions, where the relay can observe multiple replicas of the transmitted signals. Provided that the relay $R$ uses $D$-branch diversity, the received signal is expressed as

$$
\boldsymbol{Y}_{R}=\boldsymbol{H}_{A} X_{A}+\boldsymbol{H}_{B} X_{B}+\boldsymbol{Z}_{R}
$$

where

$$
\begin{aligned}
& \boldsymbol{Y}_{R}= {\left[\begin{array}{c}
Y_{R}(0) \\
Y_{R}(1) \\
\vdots \\
Y_{R}(D-1)
\end{array}\right], \boldsymbol{H}_{A}=\left[\begin{array}{c}
H_{A}(0) \\
H_{A}(1) \\
\vdots \\
H_{A}(D-1)
\end{array}\right], } \\
& \boldsymbol{H}_{B}=\left[\begin{array}{c}
H_{B}(0) \\
H_{B}(1) \\
\vdots \\
H_{B}(D-1)
\end{array}\right], \boldsymbol{Z}_{R}=\left[\begin{array}{c}
Z_{R}(0) \\
Z_{R}(1) \\
\vdots \\
Z_{R}(D-1)
\end{array}\right] .
\end{aligned}
$$

Here, $Y_{R}(d), H_{A}(d), H_{B}(d)$ and $Z_{R}(d)$ denote the received signal, the channel gains from $A$ and $B$, and the Gaussian noise at the $d$-th diversity branch, respectively. Our proposed optimization method in Section III requires two parameters $\gamma$ and $\theta$ (or equivalently, one complex value of $H_{B} / H_{A}$ ) to calculate squared distances for the single-branch reception (no diversity). In the following, we will see an interesting finding:
We only need one more real-valued parameter $\eta$ for multiplebranch diversity reception despite the fact that there are $D$ times more parameters to describe the channel condition.

In our design approach, we need to compute the squared distance between all the possible points $\left(S_{A}, S_{B}\right)=\left(s_{1}, s_{2}\right)$ and $\left(s_{1}^{\prime}, s_{2}^{\prime}\right)$ for $\left(s_{1}, s_{2}\right) \neq\left(s_{1}^{\prime}, s_{2}^{\prime}\right) \in \mathbb{Z}_{4} \times \mathbb{Z}_{4}$. For multiplebranch diversity reception, the squared distance becomes

$$
\begin{aligned}
& d_{\left(s_{1}, s_{2}\right)-\left(s_{1}^{\prime}, s_{2}^{\prime}\right)}^{2}=\left\|\boldsymbol{H}_{A} \Delta\left(s_{1}, s_{1}^{\prime}\right)+\boldsymbol{H}_{B} \Delta\left(s_{2}, s_{2}^{\prime}\right)\right\|^{2} \\
& =\left\|\left[\begin{array}{ll}
\boldsymbol{H}_{A} & \boldsymbol{H}_{B}
\end{array}\right]\left[\begin{array}{l}
\Delta\left(s_{1}, s_{1}^{\prime}\right) \\
\Delta\left(s_{2}, s_{2}^{\prime}\right)
\end{array}\right]\right\|^{2} \\
& =\|\boldsymbol{Q} \boldsymbol{R} \boldsymbol{\Delta}\|^{2}=\|\boldsymbol{R} \boldsymbol{\Delta}\|^{2},
\end{aligned}
$$

where $\boldsymbol{Q} \in \mathbb{C}^{D \times 2}$ and $\boldsymbol{R} \in \mathbb{C}^{2 \times 2}$ denote the left unitary matrix and the upper-triangular matrix, both of which can be given by the QR decomposition of a compound channel matrix $\left[\boldsymbol{H}_{A}, \boldsymbol{H}_{B}\right]$. Here, we let $\boldsymbol{\Delta}=\left[\Delta\left(s_{1}, s_{1}^{\prime}\right), \Delta\left(s_{2}, s_{2}^{\prime}\right)\right]^{\mathrm{T}}$, where $[\cdot]^{\mathrm{T}}$ denotes the transpose. The $\mathrm{QR}$ decomposition is very useful to reduce the required parameters. The uppertriangular matrix $\boldsymbol{R}$ can be expressed as

$$
\boldsymbol{R}=\alpha\left[\begin{array}{cc}
1 & \gamma \exp (\jmath \theta) \\
0 & \eta
\end{array}\right],
$$

where $\alpha, \gamma, \theta$ and $\eta$ are non-negative real values. For instance in two-branch diversity, these are written as $\alpha=\sqrt{\left|H_{A}(0)\right|^{2}+\left|H_{A}(1)\right|^{2}}, \quad \gamma=\mid H_{A}^{*}(0) H_{B}(0)+$ $H_{A}^{*}(1) H_{B}(1) \mid / \alpha^{2}, \theta=\angle\left(H_{A}^{*}(0) H_{B}(0)+H_{A}^{*}(1) H_{B}(1)\right)$, and $\eta=\left|H_{A}(0) H_{B}(1)-H_{A}(1) H_{B}(0)\right| / \alpha^{2}$, where [.]* being the complex conjugate. Using the parameters, we obtain

$$
\begin{aligned}
& d_{\left(s_{1}, s_{2}\right)-\left(s_{1}^{\prime}, s_{2}^{\prime}\right)}^{2}= \\
& \alpha^{2}\left(\left|\Delta\left(s_{1}, s_{1}^{\prime}\right)+\gamma \mathrm{e}^{\jmath \theta} \Delta\left(s_{2}, s_{2}^{\prime}\right)\right|^{2}+\eta^{2}\left|\Delta\left(s_{2}, s_{2}^{\prime}\right)\right|^{2}\right) .
\end{aligned}
$$

Since the first term is equivalent to the single-branch case, see (5), the chief difference between the single-branch and multiple-branch denoising lies in the last term including $\eta$. It implies that we just need to select the best maps according to the three channel parameters $\gamma, \theta$ and $\eta$. Note that the absolute value of $\alpha$ is not required for the design procedure. 


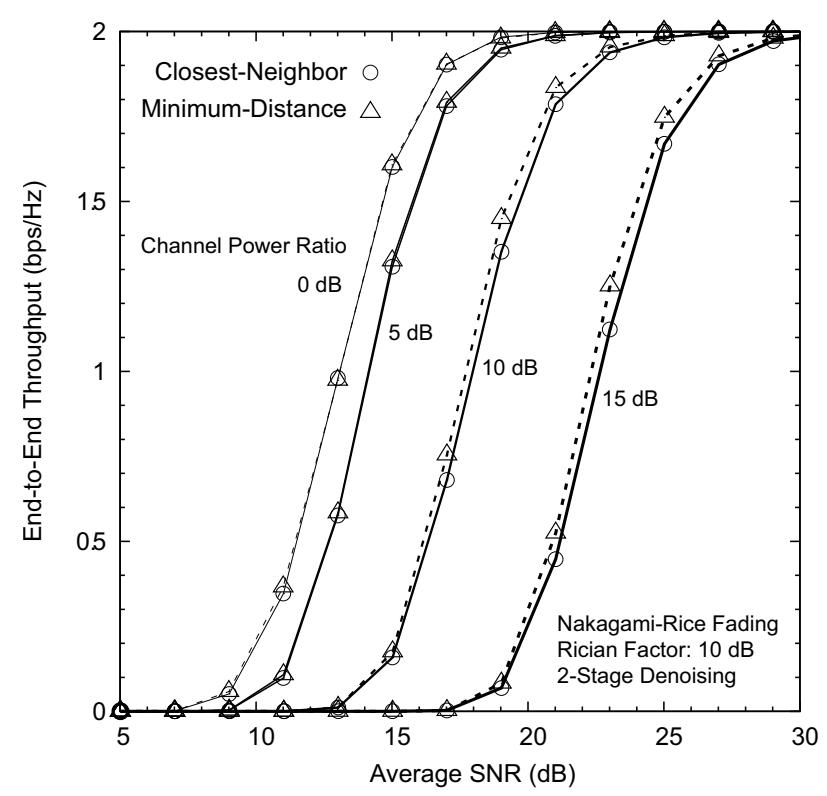

(a) $10 \mathrm{~dB}$ Rician factor

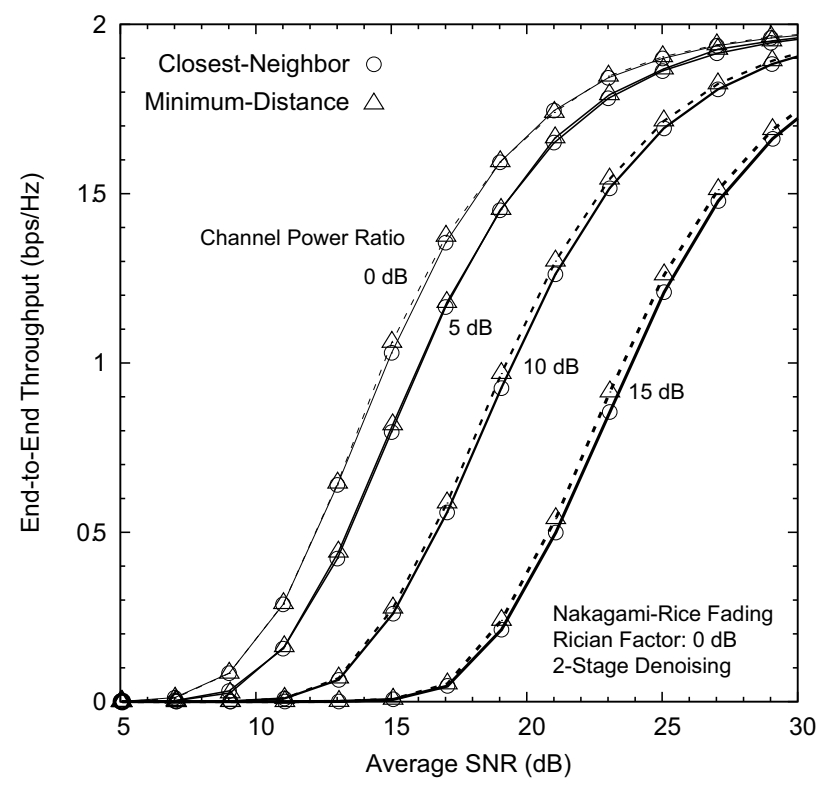

(b) $0 \mathrm{~dB}$ Rician factor

Fig. 12. Performance comparison between closest-neighbor clustering and minimum-distance criterion in end-to-end throughput as a function of average SNR in Nakagami-Rice fading channels. (2-stage denoising, QPSK used at the MA stage)

The additional parameter $\eta$ shortens the radii of the circular boundaries of selection rules in Figs. 4 and 10 from 1 to $\sqrt{1-\eta^{2}}$. When the channel condition yields $\eta \geq 1 / \sqrt{2}$, some assignment regions are eliminated. This implies that the diversity reception can reduce the probability of occurrence of undesirable singular points, as well as the severe signal fading. When the diversity effect is significant, we have $\eta \geq 1$ and the 5-ary denoising is no longer useful, but we can rely solely on the pure XOR denoising for reliable relaying.

\section{Performance Evaluation for Extended System Scenarios}

In this section, we present performance results for the extensions discussed above. We first show the performance comparison between the closest-neighbor clustering and the minimum-distance criterion in Figs. 12 (a) and (b), respectively for a Rician factor of $10 \mathrm{~dB}$ and $0 \mathrm{~dB}$. The figures plot the end-to-end throughput performance of 5-ary denoising for two-stage QPSK bidirectional relaying. The closest-neighbor clustering uses ten best codes dependent on Fig. 4, while the minimum-distance criterion uses six best codes according to Fig. 10. The results suggest that the minimum-distance criterion is slightly better than the closest-neighbor clustering, especially for a large channel power ratio $\mathbb{E}\left[\left|H_{A}\right|^{2}\right] / \mathbb{E}\left[\left|H_{B}\right|^{2}\right]$. It is because the minimum-distance criterion does not decrease the minimum distance at the MA stage and makes priority use of QPSK rather than 5QAM at the BC stage. This results in more reliable communications at the $\mathrm{BC}$ stage without sacrificing the reliability at the MA stage.

The end-to-end throughput performance for the case of 16QAM bidirectional relaying systems is plotted in Figs. 13 (a) and (b), for a Rician factor of $10 \mathrm{~dB}$ and $0 \mathrm{~dB}$, respectively. The channel follows a frequency-flat NakagamiRice fading. The curves denoted as "XOR denoising," "16-ary denoising," "optimum denoising" and "16-ary w/ precoding" represent the systems using the pure XOR operation at the relay, the adaptive network coding with 16-ary cardinality (16 codes), the adaptive network coding with all the best codes (about 400 codes), and the 16-ary denoising for a phase-synchronizing precoding ( 7 codes), respectively. For the irregular network codes with more-than 16 cardinality, we use well-designed signal constellations that are individually optimized by using the sphere packing approach (a detailed design method can be seen in Appendix). As shown in these figures, the precoding technique offers a good performance with the minimum available cardinality. It is expected that the throughput can be further increased when we can use an amplitude control as well as the phase control. However, when such a precoding technique is not available for the distributed terminals, the simple XOR operation (constant 1 code) suffers from a severe performance degradation due to the frequently occurred distance shortening as depicted in Fig. 11. One of our proposed schemes with 16-ary denoising (adaptive 16 network codes) can make a significant performance improvement. Furthermore, the proposed scheme with optimized denoising (adaptive 400 codes whose cardinality spreads from 16 through 29) can dramatically improve the throughput at the slight cost of the increased cardinality. Such a substantial performance gain comes from the avoidance of the singular points as illustrated in Figs. 11 (a), (b) and (c).

Finally, we evaluate the impact that the diversity reception has on the end-to-end throughput performance. Figs. 14 (a) and (b) show the throughput curves versus the average SNR 


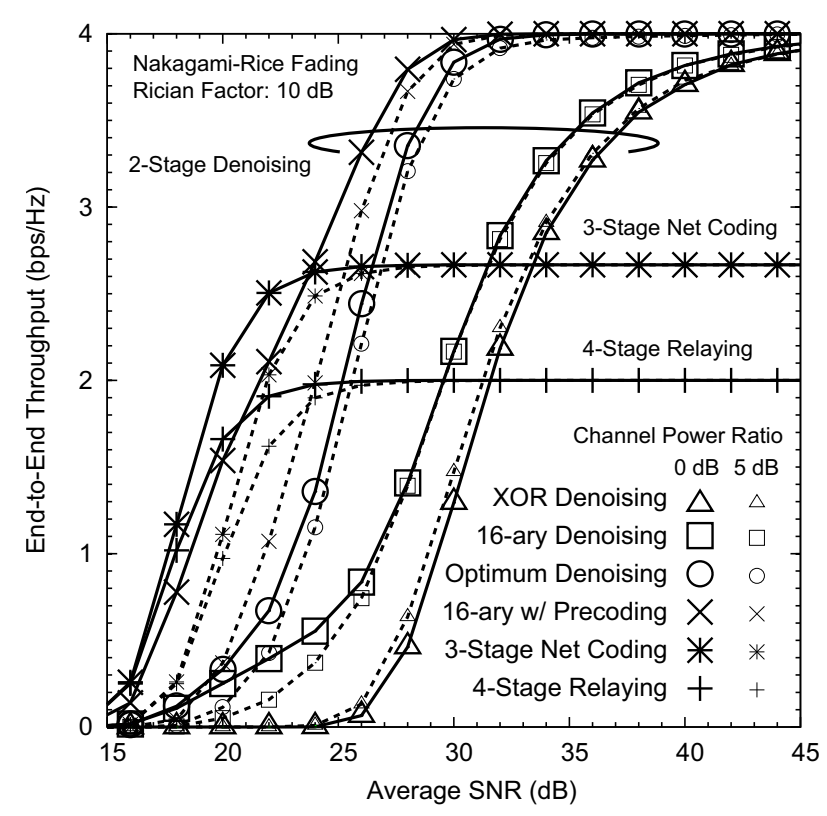

(a) $10 \mathrm{~dB}$ Rician factor

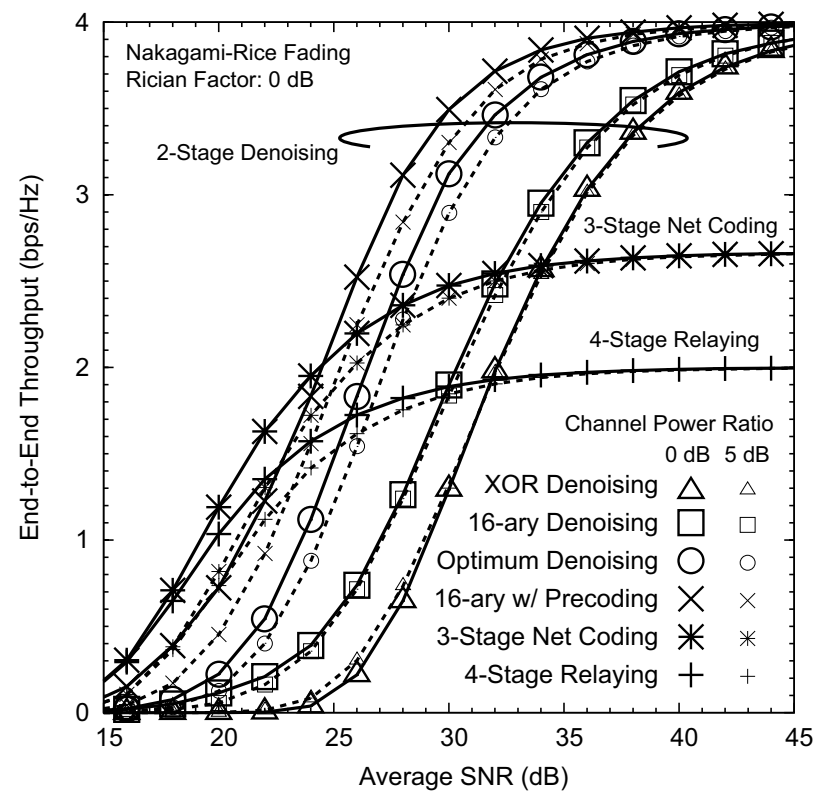

(b) $0 \mathrm{~dB}$ Rician factor

Fig. 13. Performance of 16QAM relaying in end-to-end throughput as a function of average SNR in Nakagami-Rice fading channels.

for frequency-selective Nakagami-Rice fading channels, with a Rician factor of $10 \mathrm{~dB}$ and $0 \mathrm{~dB}$, respectively (QPSK is used at the MA stage). We assume that the channel delay power profile is an exponential decaying 3-path fading with a certain decay parameter. All the terminals employ the optimum equalizer to deal with the delayed waves. These figures depict that the performance advantage of 5-ary denoising against 4ary denoising becomes small when the channel approaches the equal-gain power profile due to a significant diversity effect. As mentioned before, this is because the diversity effect can considerably reduce the probability of the singular points and, therefore, the 5-ary denoising is less likely required.

\section{CONCLUSION}

In this paper, we have considered the design problem of two-way wireless relaying, in which the communication is carried out in two stages. In the first multiple access (MA) Stage, the terminals transmit simultaneously to the relay. Based on the received signals in the MA stage, in the second broadcast (BC) stage, the relay uses the denoiseand-forward (DNF) scheme to create a proper signal that is then broadcasted to the nodes. Our focus is the design of constellations and maps that represent the network coding function, performed by the relay at the physical layer. We have first discussed the scenario when QPSK is used during the MA stage. The analysis and the results have shown that, for certain channel conditions, the denoising operation at the relay should make use of unconventional 5-ary modulations to improve the throughput performance. We have illustrated how such a 5QAM constellation should be designed and we have devised a method for adaptive selection of the network coding function, where the adaptation is exhibited with respect to the varying channel conditions. Through performance evaluations, it has been demonstrated that the proposed denoising scheme significantly improves the achievable end-to-end throughput, especially for Nakagami-Rice fading channels. The proposed scheme has a practical significance, as it does not require any phase control over all the distributed terminals.

Furthermore, we have discussed the design strategy in several ways: A simplified criterion of adaptive network coding is introduced to improve reliability at the BC stage. It is shown that this criterion is useful when the constellations used at the MA stage are of higher order (16QAM), because it reduces the space of network codes that are used in the selection process. Finally, we have considered the scenario in which the relay exploits multiple-branch diversity, and we have derived the optimizing function that is used to design denoising maps. Our results have revealed that the performance gain of 5-ary denoising becomes small because the distance shortening can be compensated by the diversity effect.

A natural topic to be considered in future is the design strategy for adaptive modulation and coding based on the channel conditions in DNF two-way relaying systems.

\section{ACKNOWLEDGMENT}

This work is partially supported by JSPS Postdoctoral Fellowships for Research Abroad, TAF, SCAT Japan, and the Danish Research Council for Technology and Production.

\section{APPENDIX}

Let $p_{s}$ be the a priori probability of $s=\mathcal{C}\left(\hat{S}_{A}, \hat{S}_{B}\right)$. The best 5 -ary network codes have $p_{0}=1 / 4$ and $p_{1}=p_{2}=p_{3}=$ $p_{4}=3 / 16$. Therefore, the sphere radius for $s=0$ should 


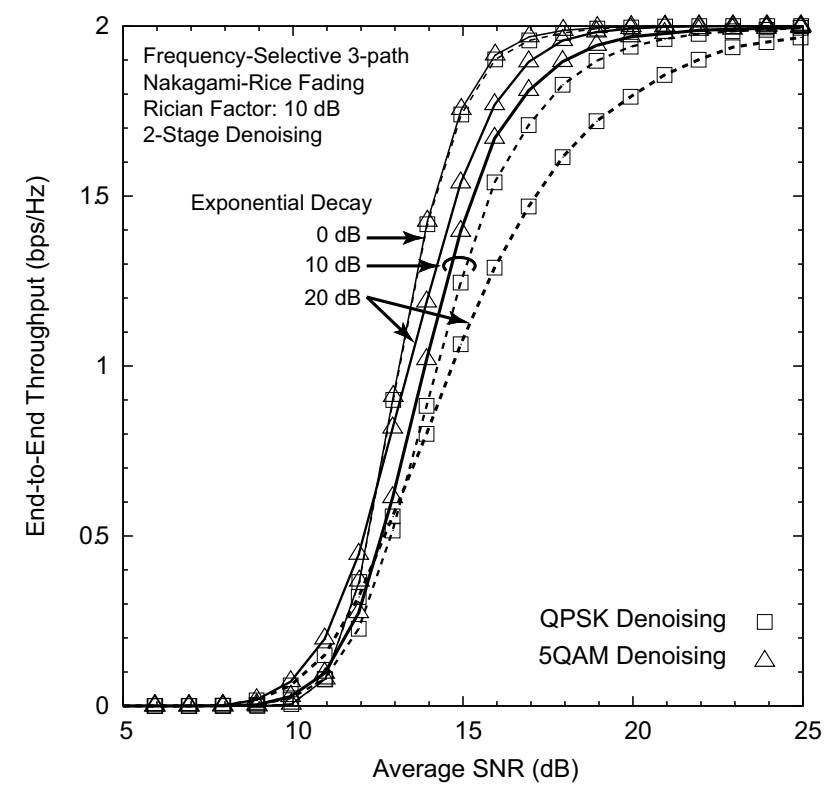

(a) $10 \mathrm{~dB}$ Rician factor

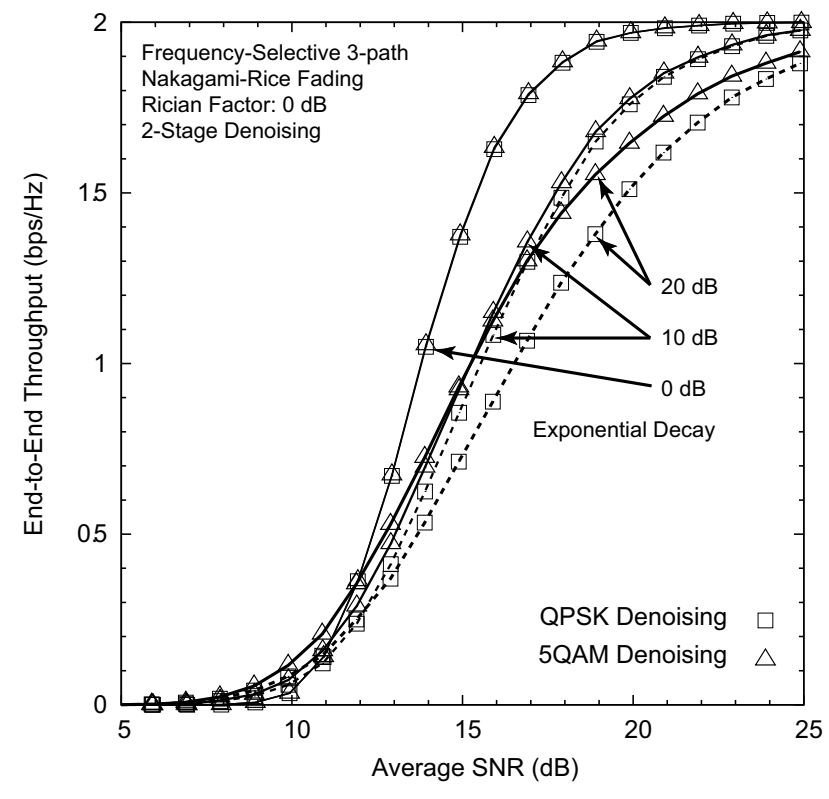

(b) $0 \mathrm{~dB}$ Rician factor

Fig. 14. Effect of diversity reception in end-to-end throughput as a function of average SNR in frequency-selective 3-path Nakagami-Rice fading channels with exponential decaying profile. (2-stage denoising, QPSK used at the MA stage)

be larger than the others. For optimizing a constellation, we consider a ring-shaped modulation, in which the constellation points $\left\{\chi_{i}\right\}$ are located as follows:

$$
\begin{gathered}
\chi_{0}=0, \chi_{1}=\left(R_{1}+R_{2}\right) \mathrm{e}^{\jmath\left(\vartheta+\phi+\frac{\pi}{2}\right)}, \chi_{2}=\frac{R_{2}}{\sin \vartheta} \mathrm{e}^{\jmath(\vartheta+\pi / 2)}, \\
\chi_{3}=\frac{R_{2}}{\sin \vartheta} \mathrm{e}^{-\jmath \vartheta-\pi / 2)}, \quad \chi_{4}=\left(R_{1}+R_{2}\right) \mathrm{e}^{-\jmath(+\phi-\pi / 2)},
\end{gathered}
$$

where we can express

$$
\begin{aligned}
& R_{1}^{2}=\epsilon+\frac{1}{\rho} \ln \frac{1}{4}, \quad R_{2}^{2}=\epsilon+\frac{1}{\rho} \ln \frac{3}{16}, \\
& \cos \phi=\frac{\left(R_{1}+R_{2}\right)^{2}+R_{2}^{2} / \sin ^{2} \vartheta-4 R_{2}^{2}}{2\left(R_{1}+R_{2}\right) R_{2} / \sin \vartheta} .
\end{aligned}
$$

The value $\epsilon$ denotes some sort of log-likelihood that indicates a reliability achieved by the given radii. The notation $\rho$ stands for an SNR at the receiver. Here, we optimize the values of $\vartheta$, $\phi, R_{1}$ and $R_{2}$ to obtain the best constellation. The centralized energy of this constellation is written as

$$
\begin{aligned}
E_{0}= & \sum_{s=0}^{4} p_{s}\left|\chi_{s}\right|^{2}-\left|\sum_{s=0}^{4} p_{s} \chi_{s}\right|^{2} \\
= & \frac{3}{8}\left(\left(R_{1}+R_{2}\right)^{2}+\frac{R_{2}^{2}}{\sin ^{2} \vartheta}\right) \\
& -\frac{9}{64}\left(\frac{R_{2}}{\tan \vartheta}+\left(R_{1}+R_{2}\right) \cos (\vartheta+\phi)\right)^{2} .
\end{aligned}
$$

This energy $E_{0}$ is a decreasing function in terms of $\vartheta$. Hence, the optimal value of $\vartheta$ minimizing $E_{0}$ satisfies $\sin \vartheta=$
$R_{2} /\left(R_{1}+R_{2}\right)$, and the minimized energy is rewritten as

$$
\begin{aligned}
& E_{0}=\frac{3}{16}\left(R_{1}+R_{2}\right)^{2} \\
& \quad\left(4-3\left(1-\frac{R_{2}^{2}}{\left(R_{1}+R_{2}\right)^{2}}\right)\left(1-\frac{2 R_{2}^{2}}{\left(R_{1}+R_{2}\right)^{2}}\right)^{2}\right) .
\end{aligned}
$$

Plugging (15) into the above equation, we can solve the allowable likelihood $\epsilon$ as a function of $E_{0}$ and $\rho$. When we take a bound $E_{0} \leq 1$, we can obtain the likelihood bound $\epsilon$ and the optimal radii $R_{1}$ and $R_{2}$, numerically.

Assuming $\sigma^{2} \ll 1$, we can obtain a very tight solution analytically. This assumption gives $\left(R_{1}+R_{2}\right)^{2} \simeq 2\left(R_{1}^{2}+\right.$ $\left.R_{2}^{2}\right)=4 R_{2}^{2}+2 \rho^{-1} \ln \frac{4}{3}$, and we obtain the following cubic equation from (19):

$$
\begin{gathered}
165 X^{3}+(216 \beta-64) X^{2}+ \\
\left(81 \beta^{2}-64 \beta\right) X+\left(6 \beta^{3}-16 \beta^{2}\right)=0,
\end{gathered}
$$

where $X=R_{2}^{2}$ and $\beta=\rho^{-1} \ln 4 / 3$. Since the cubic function has analytical solution, we can calculate the optimum parameters. It is confirmed that the analytical solution with the approximation matches well the exact solution.

For any arbitrary network coding, the sphere radii should be optimized according to the a priori probability and the SNR at the receiver. However, the designed constellation with optimized radii only provides a visible advantage against the optimized constellation with uniform radii for a very low SNR regime. In order to design all the best 400 network codes whose cardinality lies between 16 and 29 for 16QAM two-way relaying, we focus on the constellation pattern with uniform radii. In this paper, we propose a design method based on a greedy sphere packing described in Algorithm 3. 


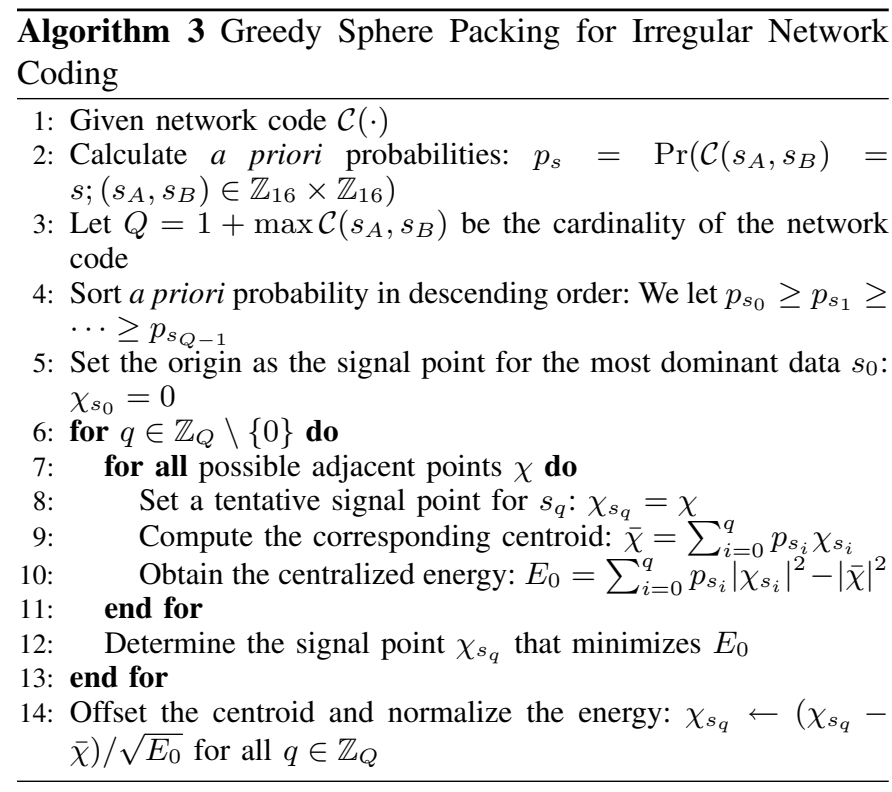

\section{REFERENCES}

[1] T. Koike-Akino, P. Popovski, and V. Tarokh, "Denoising maps and constellations for wireless network coding in two-way relaying systems," IEEE GLOBECOM, New Orleans, Nov.-Dec. 2008.

[2] R. Ahlswede, N. Cai, S.-Y. R. Li, and R. W. Yeung, "Network information flow," IEEE Trans. Inf. Theory, vol. IT-46, no. 4, pp. 1204-1216, July 2000.

[3] Y. Wu, P. A. Chou, and S.-Y. Kung, "Information exchange in wireless networks with network coding and physical-layer broadcast," Microsoft, Tech. Rep. MSR-TR-2004-78, Aug. 2004.

[4] P. Larsson, N. Johansson, and K.-E. Sunell, "Coded bi-directional relaying," Scandinavian Workshop ADHOC'05, May 2005.

[5] P. Larsson, N. Johansson, and K.-E. Sunell, "Coded bi-directional relaying," IEEE VTC-Spring, vol. 2, pp. 851-855, May 2006.

[6] P. Popovski and H. Yomo, "Bi-directional amplification of throughput in a wireless multi-hop network," IEEE VTC-Spring, May 2006.

[7] S. Katti, D. Katabi, W. Hu, H. Rahul, and M. Médard, "The importance of being opportunistic: Practical network coding for wireless environments," A. Allerton Conf. Commun., Control, and Comput., Sept. 2005.

[8] S. Katti, H. Rahul, W. Hu, D. Katabi, M. Médard, and J. Crowcroft, "XORs in the air: practical wireless network coding," Conf. Applications, Technol., Architect., and Protocols Comput. Commun., Sept. 2006.

[9] S. Katti, S. Gollakota, and D. Katabi, "Embracing wireless interference: Analog network coding," ACM SIGCOMM 2007, Aug. 2007.

[10] C. E. Shannon, "Two-way communications channels," 4th Berkeley Symp. Math. Stat. Prob., Chicago, IL, pp. 611-644, June 1961.

[11] T. Cover and J. A. Thomas, Elements of Information Theory, Second Edition, John Wiley \& Sons, 2006.

[12] S. J. Kim, N. Devroye, P. Mitran, and V. Tarokh, "Comparison of bidirectional relaying protocols," IEEE Sarnoff Symposium, Apr. 2008.

[13] S. J. Kim, P. Mitran, and V. Tarokh, "Performance bounds for bidirectional coded cooperation protocols," IEEE Trans. Inf. Theory, vol. 54, no. 11, pp. 5235-5241, Nov. 2008

[14] P. Popovski and H. Yomo, "Physical network coding in two-way wireless relay channels," IEEE ICC, Glasgow, Scotland, June 2007.

[15] P. Popovski and H. Yomo, "The anti-packets can increase the achievable throughput of a wireless multi-hop network," IEEE ICC, June 2006

[16] B. Rankov and A. Wittneben, "Achievable rate regions for the two-way relay channel," IEEE ISIT, pp. 1688-1672, July 2006.

[17] J. H. Conway and N. J. A. Sloane, Sphere Packings, Lattices and Groups, Springer, Dec. 1998.

[18] B. Rankov and A. Wittneben, "Spectral efficient protocols for halfduplex fading relay channels," IEEE J. Select. Areas Commun., vol. 25, no. 2, pp. 379-389, Feb. 2007.

[19] S. Zhang, S. C. Liew, and P. P. Lam, "Physical-Layer Network Coding," ACM MOBICOM 2006, Los Angeles, Sept. 2006.

[20] T. J. Oechtering, C. Schnurr, I. Bjelakovic, and H. Boche, "Broadcast capacity region of two-phase bidirectional relaying," IEEE Trans. Inf. Theory, vol. 54, no. 1, pp. 454-458, Jan. 2008.
[21] K. Narayanan, M. P. Wilson, and A. Sprintson, "Joint physical layer coding and network coding for bi-directional relaying," Allerton Conference on Communication, Control and Computing, Monticello, IL, USA, 2007.

[22] B. Nazer and M. Gastpar, "Computation over multiple access channels," IEEE Trans. Inf. Theory, vol. 53, no. 10, pp. 3498-3516, Oct. 2007.

[23] P. Mitran, "The diversity-multiplexing tradeoff for independent parallel MIMO channels," IEEE ISIT, Toronto, July 2008.

[24] E. Fasolo, F. Rossetto, and M. Zorzi, "Network coding meets MIMO" NetCod 2008, Hong Kong, Jan. 2008.

[25] T. Koike-Akino, P. Popovski, and V. Tarokh, "Denoising strategy for convolutionally-coded bidirectional relaying," IEEE ICC 2009, Dresden, Germany, June 2009.



Toshiaki Koike-Akino received the B.S. degree in electrical and electronics engineering, M.S. and Ph.D. degrees in communications and computer engineering from Kyoto University in 2002, 2003 and 2005 , respectively. Since 2006 , he has been a postdoctoral researcher in Harvard University. His research interest includes digital signal processing for cooperative communications. He has been a JSPS Postdoctoral Fellow for Research Abroad since 2008. He received the YRP Encouragement Award 2005, the 21st TELECOM System Technology Award, the 2008 Ericsson Young Scientist Award, the IEEE GLOBECOM'08 Best Paper Award in Wireless Communications Symposium, and the 24th TELECOM System Technology Encouragement Award,

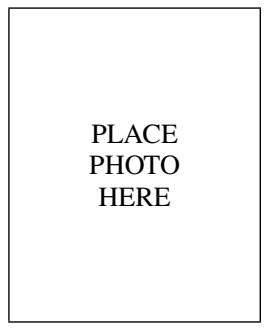

Petar Popovski received the Dipl.-Ing. in electrical engineering and M.Sc. in communication engineering from the Faculty of Electrical Engineering, Sts. Cyril and Methodius University, Skopje, Macedonia, in 1997 and 2000, respectively and a Ph.D. degree from Aalborg University, Denmark, in 2004. He currently holds two positions, the first as Assistant Professor at the Department of Electronic Systems at Aalborg University and the second as a wireless architect at Oticon A/S. He has more than 70 publications in journals, conference proceedings and books and has more than 20 patents and patent applications. He has served as a technical program committee member in various conferences and workshops, including IEEE SECON, IEEE ICCCN, IEEE PIMRC, IEEE GLOBECOM, etc. Dr. Popovski has been a Guest Editor for special issues in EURASIP Journal on Applied Signal Processing and the Journal of Communication Networks. He is editor in Ad Hoc and Sensor Wireless Networks journal and editor in IEEE Communications Letters. In January 2009 he received the Young Elite Researcher award from the Danish Ministry of Science and Technology. His research interests are in the broad area of wireless communication and networking, information theory and protocol design.

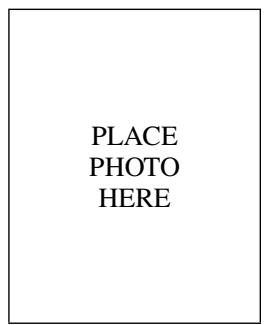

Vahid Tarokh worked at AT\&T Labs-Research and AT\&T wireless services until August 2000, where he was the head of the Department of Wireless Communications and Signal Processing. In September 2000, he joined Department of Electrical Engineering and Computer Sciences (EECS) at MIT as an associate professor. In June 2002, he joined Harvard University as a Gordon McKay Professor of Electrical Engineering. Since July 2005, he is a Hammond Vinton Hayes Senior Fellow of Electrical Engineering at Harvard University, and a Perkins professor. His research is mainly focused in the areas of signal processing, communications (wireline and wireless) and networking. He has received a number of awards and holds 2 honourary degrees. 